\title{
Temporal Firing Patterns of Purkinje Cells in the Cerebellar Ventral Paraflocculus During Ocular Following Responses in Monkeys II. Complex Spikes
}

\author{
YASUSHI KOBAYASHI, ${ }^{1,2}$ KENJI KAWANO,,${ }^{3,4}$ AYA TAKEMURA, ${ }^{3}$ YUKA INOUE, ${ }^{3}$ TOSHIHIRO KITAMA, ${ }^{3}$ \\ HIROAKI GOMI, ${ }^{4,5}$ AND MITSUO KAWATO ${ }^{1}$ \\ ${ }^{1}$ ATR Human Information Processing Research Laboratories, Kyoto 619-0288; ${ }^{2} J S T$-CREST, Aichi 444-8585; \\ ${ }^{3}$ Neuroscience Section, Electrotechnical Laboratory, Ibaraki 305-8568; ${ }^{4}$ JST-CREST, Ibaraki 305-8568; and ${ }^{5}$ NTT Basic \\ Research Laboratories, Nippon Telegraph and Telephone Corporation, Kanagawa 243-0198, Japan
}

Kobayashi, Yasushi, Kenji Kawano, Aya Takemura, Yuka Inoue, Toshihiro Kitama, Hiroaki Gomi, and Mitsuo Kawato. Temporal firing patterns of Purkinje cells in cerebellar ventral paraflocculus during ocular following responses in monkeys. II. Complex spikes. J. Neurophysiol. 80: 832-848, 1998. Many theories of cerebellar motor learning propose that complex spikes (CS) provide essential error signals for learning and modulate parallel fiber inputs that generate simple spikes (SS). These theories, however, do not satisfactorily specify what modality is represented by CS or how information is conveyed by the ultra-low CS firing rate $(1 \mathrm{~Hz})$. To further examine the function of CS and the relationship between CS and SS in the cerebellum, CS and SS were recorded in the ventral paraflocculus (VPFL) of awake monkeys during ocular following responses (OFR). In addition, a new statistical method using a generalized linear model of firing probability based on a binomial distribution of the spike count was developed for analysis of the ultra-low CS firing rate. The results of the present study showed that the spatial coordinates of CS were aligned with those of SS and the speed-tuning properties of CS and SS were more linear for eye movement than retinal slip velocity, indicating that CS contain a motor component in addition to the sensory component identified in previous studies. The generalized linear model to reproduce firing probability confirmed these results, demonstrating that CS conveyed high-frequency information with its ultra-low firing frequency and conveyed both sensory and motor information. Although the temporal patterns of the CS were similar to those of the SS when the sign was reversed and magnitude was amplified $\sim 50$ times, the velocity/acceleration coefficient ratio of the eye movement model, an aspect of the CS temporal firing profile, was less than that of the SS, suggesting that CS were more sensory in nature than SS. A cross-correlation analysis of SS that are triggered by CS revealed that short-term modulation, that is, the brief pause in SS caused by CS, does not account for the reciprocal modulation of SS and CS. The results also showed that three major aspects of the CS and SS individual cell firing characteristics were negatively correlated on a cell-to-cell basis: the preferred direction of stimulus motion, the mean percent change in firing rate induced by upward stimulus motion, and patterns of temporal firing probability. These results suggest that CS may contribute to long-term interactions between parallel and climbing fiber inputs, such as long-term depression and/or potentiation.

\section{INTRODUCTION}

A remarkable feature of the Purkinje cells in the cerebellum is that each receives two major afferents that differ dramatically in their firing dynamics: multiple parallel fiber inputs that generate simple spikes ( $\mathrm{SS}$ ) at rates up to several hundred discharges per second and a single climbing fiber input that generates complex spikes (CS) at rates that do not exceed more than a few discharges per second (Thach 1968). The type of information transmitted by the ultra-low CS firing rate and the effect of a signal with such low temporal resolution on the cerebellum are still not completely resolved.

The present study quantitatively examines CS function by examining CS and SS responses in the ventral paraflocculus (VPFL) during ocular following responses (OFR) in awake monkeys (Miles et al. 1986) and quantifying the relation between the two discharges and the retinal slip and the eye movement. Recent modeling studies of the temporal firing profiles of SS during OFR using an inverse-dynamics model (a linear combination of eye acceleration, velocity, and position) demonstrated that SS in the VPFL encode dynamic motor commands (Gomi et al. 1998; Kawano et al. 1996; Shidara et al. 1993). In the present study, in addition to the electrophysiological experiments, this model has been extended to a more sophisticated generalized linear model (Kawato 1995) to analyze the correlation between the ultralow CS firing rate and the motor commands or retinal slip.

OFR are tracking movements of the eyes evoked by movements of a visual scene and are thought to be important for the visual stabilization of gaze. It was advantageous for several reasons to study CS function by recording them in the VPFL during OFR. The OFR are primarily under negative feedback control because this behavior is primarily in response to retinal slip, which is the difference between the image motion and the eye movement. The early phase of the OFR, however, is controlled in an open-loop manner, and this early phase has been shown to be subject to longterm adaptive modification by visual error signals (Miles and Kawano 1986). OFR are reflexes induced by the retinal slip, so it is technically easy to obtain a large number of trials, thus increasing the signal-to-noise ratio. It is possible to quantify the correlation between the sensory error signal (retinal slip) and CS firing because it is possible to accurately control the parameters of the visual stimulus. SS evoked during OFR have been recorded and characterized in Purkinje cells in the VPFL (Gomi et al. 1998; Kawano et al. 1996; Shidara and Kawano 1993; Shidara et al. 1993). 
The climbing fiber projections from the inferior olive (IO) to the VPFL have been well characterized (Gerrits and Voogd 1982, 1989; Langer et al. 1985). In the rabbit flocculus, a large number of CS are evoked by movement of a large visual stimulus (Graf et al. 1988; Simpson and Alley 1974). CS also were recorded in the VPFL of monkeys during tracking of a small target (Stone and Lisberger $1990 \mathrm{~b}$ ). Thus a considerable number of CS are expected to be evoked in the VPFL during OFR.

The CS in the VPFL have previously been well characterized during smooth pursuit eye movement by Stone and Lisberger (1990b), who concluded that CS were driven by contralaterally or upward directed image motion. CS were modulated out-of-phase with SS. By spike-triggered averaging analysis, they concluded that CS during steady-state pursuit were driven by the retinal slip associated with imperfect pursuit.

In this study, we have advanced the quantitative understanding of CS during OFR in regard to the following four points and have provided critical data to examine the major theories of CS function. First, we developed a new statistical method for quantitatively analyzing what information is encoded in the temporal patterns of CS firing rate. With this new technique, we have demonstrated that the CS firing probability carries very high-frequency temporal information that matches that of the SS. Second, cell-to-cell negative correlations between the firing characteristics of SS and CS for individual cells were revealed. Third, although in previous studies the sensory aspects of CS were well studied, we have added new evidence for a motor-related nature of CS. Fourth, we examined the relationship between the velocity of eye movement or retinal slip and the firing rates of CS over a wide range of step ramp speeds.

\section{METHODS}

Our methods for preparing the monkeys, for presenting visual stimuli, and recording the simple spike of Purkinje cells ( $\mathrm{P}$ cells) are presented in detail in the preceding paper (Gomi et al. 1998). Here, we describe only those additional techniques that were used in the analysis of simple- and complex-spike responses.

\section{Visual stimuli}

Visual stimuli were designed to study the directional selectivity of neural firings (experiment 1), effects of changes in stimulus velocity on neural firings (experiment 2), and temporal patterns of firing rate and firing probability (experiment 3 ).

EXPERIMENT 1. The directional firing characteristics of 13 cells were examined by moving the stimuli in eight directions $(\theta=0$, $45,90,135,180,225,270$, and $\left.315^{\circ}\right)$ at a constant speed of $80^{\circ} \%$ $\mathrm{s}$. The stimulus was presented and moved $\geq 40$ times ( $40-77$ trials, mean $=57$ trials $)$ in each direction while recording from each cell (320-616 trials, mean $=456$ trials altogether). Because the latency of the change in SS firing rate during the OFR is $\sim 40 \mathrm{~ms}$ from the onset of stimulus motion (Shidara and Kawano 1993), the spike modulation for each stimulus direction was calculated as the mean firing rate minus the spontaneous firing rate over an interval extending from 40 to $220 \mathrm{~ms}$ after the onset of stimulus motion. Spontaneous rate was calculated as the mean firing rate over an interval from -100 to $40 \mathrm{~ms}$ after the onset of stimulus motion. The preferred direction of SS or CS of each cell was calculated as the direction of the average vector of modulation vectors for each direction, defined as a two-dimensional vector with the same direction as the stimulus motion $(\theta)$ and a length equal to the spike modulation defined above. Because we did not use a three-dimensional (3-D) planetarium projector system ( Graf et al. 1988), we cannot directly argue about the preferred axis of rotation in 3-D space from our experimental data.

EXPERIMENT 2. Stimuli moving at six or eight different velocities $\left(+80,+40,+20,-20,-40\right.$, and $-80^{\circ} \%$ s or $+80,+40,+20,+10$, $-10,-20,-40$, and $-80^{\circ} / \mathrm{s}$ ) were presented while recording from 12 cells. For each cell, the stimulus was moved either vertically or horizontally, so that it would overlap with the preferred and anti-preferred directions of SS. Upward and contralateral motion were assigned positive polarity. At least 70 trials (76-217 trials, mean $=134$ trials $)$ were performed at each stimulus velocity for each cell $(608-1,592$ trials, mean $=948$ trials $)$.

EXPERIMENT 3. Stimuli moving directly upward at $80^{\circ} / \mathrm{s}$ were presented to nine vertical axis cells ( $\mathrm{V}$ cells). For V cells, directly upward is close to the preferred direction of CS and the antipreferred direction of SS. Upward moving stimuli were presented $>300$ times $(312-901$ trials, mean $=579$ trials $)$ while recording from each cell.

For improving data reliability in the analysis of the firing characteristics of CS for each cell, we focused on obtaining a large number of trials rather than on increasing the number of recorded cells.

\section{Recording technique}

Purkinje cells were identified by the presence of SS and CS (Thach 1968). Before trial sessions, we carefully discriminated each single unit using a time-amplitude window discriminator, and we checked that CS and SS were derived from the same cell by confirming the occurrence of a brief pause of the SS after the CS (Sato et al. 1992). After the sessions, SS and CS were discriminated with a time resolution of 1 or $2 \mathrm{~ms}$ off-line using custom software, running on a SUN SPARK station, which clusters groups of spikes by amplitude, duration and wave form by principal component analysis.

\section{Generalized linear model}

In our previous studies, the SS firing rate was reproduced directly using an inverse-dynamics representation model, which is a linear weighted summation of the eye acceleration, velocity, and position (Gomi et al. 1998; Kawano et al. 1996; Shidara et al. 1993). The very low CS firing rate precludes the direct use of this method for the analysis of the CS temporal firing profile, but the firing probability rather than the firing rate itself can be modeled. The low CS firing rate highlighted the binomial nature of the spike count. The variance was not constant, invalidating the minimum-square-error method for parameter estimation, and the standard deviation had the same magnitude as the mean, rendering the correlation coefficient rather insensitive to the goodness of fit. The fluctuations in the CS firing rate were largely due to the variance of the binomial distribution. Theoretically, the firing rate (spikes/s) multiplied by the time bin ( $\mathrm{s}$ ) converges to the firing probability $p$ as the trial number $n$ goes to infinity with a standard deviation $\sqrt{p(1-p) / n}$ of the binomial distribution. The following is a typical example of a firing probability with the same order of magnitude as its standard deviation. The standard deviation 0.003 is close to the signal itself for $p=0.005$ ( 2.5 spikes/s multiplied by a $2-\mathrm{ms}$ bin) and $n=$ 500 . Thus for CS, the low value of the actual correlation coefficient and the predicted value does not necessarily mean a poor fit by the model. On the other hand, for SS, firing at 100 spikes/s, the signal (i.e., probability $p=0.2$ ) is much larger than the noise (i.e., 
the standard deviation is 0.02 ), and thus use of the correlation coefficient is valid.

We confirmed that the number of CS and SS, $X_{i}$, which accumulated within the time $t=i$ bin for $n$ trials, obeyed the following binomial distribution (Kobayashi et al. 1995)

$$
\operatorname{Pr}\left\{X_{i}=y_{i}\right\}=\left(\begin{array}{l}
n \\
y_{i}
\end{array}\right) p_{i}^{y_{i}}\left(1-p_{i}\right)^{n-y_{i}}
$$

where $y_{i}$ denotes the realized value of the stochastic variable $X_{i}$, that is, the observed spike number. $p_{i}$ is the spike occurrence probability within the time $t=i$ bin. The firing probability $p(t)$ as a function of time $t$ was modeled by the following generalized linear summation of acceleration, velocity, and position of eye movement, which is a smooth function of time

$$
\begin{gathered}
p(t)=S[M \cdot \ddot{\theta}(t+\delta)+B \cdot \dot{\theta}(t+\delta)+K \cdot \theta(t+\delta)+C] \\
S[x]=\frac{\exp x}{1+\exp x}
\end{gathered}
$$

where $\ddot{\theta}, \dot{\theta}$, and $\theta$ denote the acceleration, velocity, and position of the eye, and $M, B$, and $K$ denote their coefficients, respectively, while $\delta$ denotes the time delay between spike discharge and eye movement, and $C$ is a constant. The sigmoid function $S$ constrains $p(t)$ to values between 0 and 1 . This is a specific example of a generalized linear model (McCullagh and Nelder 1989).

The parameters other than the time delay were estimated using Fisher's scoring method by maximizing the following likelihood function, $L$ or its logarithm $l$ (the maximum likelihood method)

$$
\begin{aligned}
L(\mathbf{p} ; \mathbf{y}) & =\prod_{i=1}^{m} \operatorname{Pr}\left(X_{i}=y_{i}\right) \\
& =\prod_{i=1}^{m}\left(\begin{array}{l}
n \\
y_{i}
\end{array}\right) p_{i^{\prime}}^{y_{i}}\left(1-p_{i}\right)^{n-y_{i}} \\
l(\mathbf{p} ; \mathbf{y}) & =\sum_{i=1}^{m}\left\{y_{i} \log p_{i}+\left(n-y_{i}\right) \log \left(1-p_{i}\right)\right\}
\end{aligned}
$$

$m$ is the bin number included in one experiment. In experiment 3 , $m=126$, because all of the spike counts within a $2-m s$ bin were collected to calculate $y_{i}$ from 0 to $250 \mathrm{~ms}$ after the onset of stimulus motion; $m=756$ ( 6 velocities) or $m=1,008$ ( 8 velocities) in experiment 2 because stimuli with different velocities produced different sets of data. The time delay leading to the maximum likelihood was globally searched at every 2-ms step from -20 to $20 \mathrm{~ms}$.

Let $L_{1}$ denote the likelihood evaluated by the maximum likelihood estimator in $p_{i}\left(\tilde{p}_{i}=y_{i} / n\right)$, which is the best possible model but with a large degree of freedom $m$, while $L_{0}$ denotes the maximum likelihood of the current model. If the model is good, the likelihood ratio $\left(\lambda=L_{0} / L_{1}\right)$ is close to 1 , but if the model is poor, the ratio approaches 0 . The deviance $D,-2 \log \lambda$ expressed in the following equation is always positive and approaches zero as the fit becomes better and becomes large as the fit becomes poorer

$$
\begin{aligned}
D(\mathbf{y} ; \hat{\mathbf{p}}) & =2 l(\tilde{\mathbf{p}} ; \mathbf{y})-2 l(\hat{\mathbf{p}} ; \mathbf{y}) \\
& =2 \sum_{i=1}^{m}\left\{y_{i} \log \frac{y_{i}}{n \hat{p}_{i}}+\left(n-y_{i}\right) \log \frac{n-y_{i}}{n-n \hat{p}_{i}}\right\}
\end{aligned}
$$

Generally, a smaller deviance indicates a better fit. Because the deviance increases in proportion to $m$, the deviances in experiment 2 were divided by 6 or 8 (the number of different stimulus velocities) for comparison with that of experiment 3 in Table 1.

To further examine the sensory and motor characteristics of CS and SS, we compared the ability of a model based on the sensory error and the eye movement to reproduce the firing probability. Thus the model was based on a generalized linear combination of
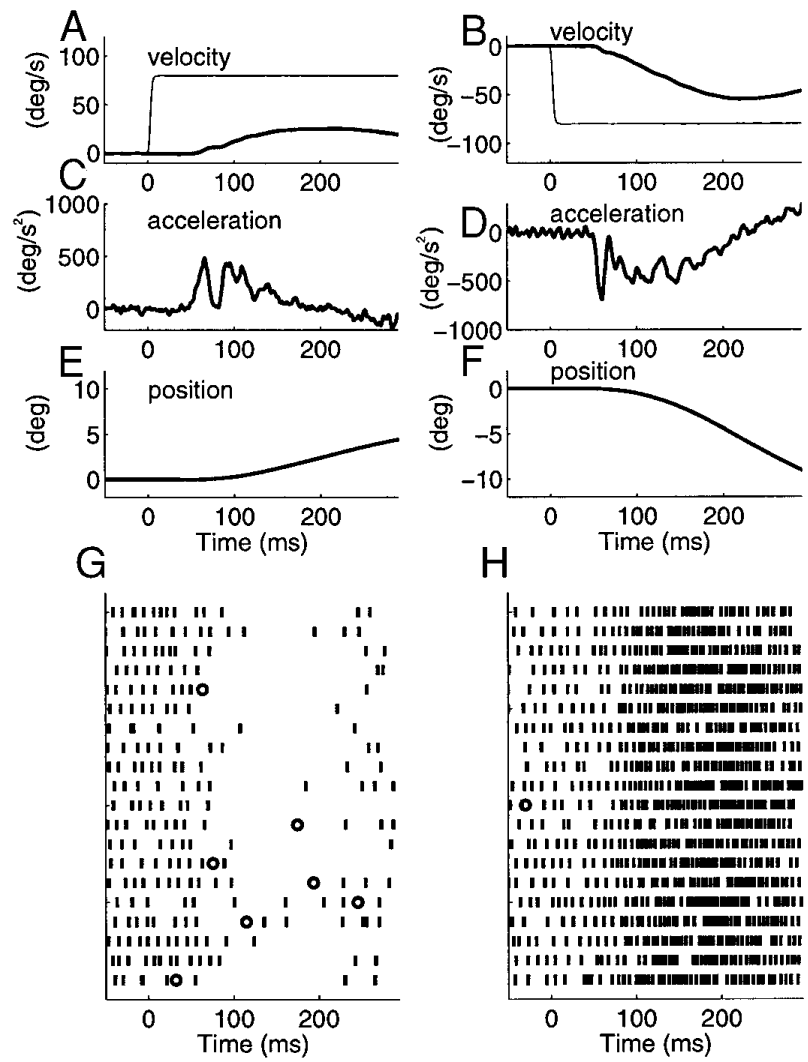

$H$

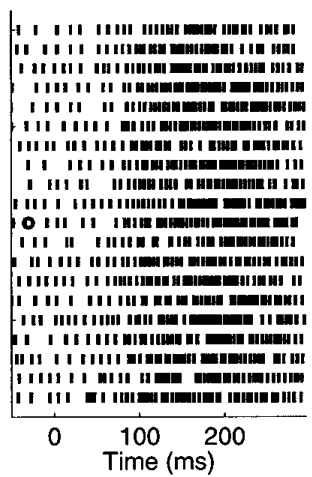

FIG. 1. Eye movements and complex spikes (CS) and simple spikes (SS) during ocular following responses (OFR). Left: responses to $80^{\circ} / \mathrm{s}$ upward stimulus motion. Right: responses to $80^{\circ}$ /s downward stimulus motion. Stimulus velocity (thin line in $A$ and $B$ ) and eye velocity (thick line in $A$ and $B$ ), eye acceleration ( $C$ and $D$ ), and eye position ( $E$ and $F$ ) as functions of time after the onset of stimulus motion during OFR are shown. $G$ and $H$ : examples of rastergrams of the SS and CS responses in a V cell during OFR to 20 presentations of upward $(G)$ and downward $(H)$ test ramps. Bars represent SS and the circles represent CS.

the acceleration, velocity, and the position of the retinal slip as well (the difference between stimulus position and eye position)

$$
p(t)=S\left[M_{r} \cdot \ddot{r}(t-\Delta)+B_{r} \cdot \dot{r}(t-\Delta)+K_{r} \cdot r(t-\Delta)+C_{r}\right]
$$

where $\ddot{r}, \dot{r}, r, C_{\mathrm{r}}$, and $\Delta$ denote the acceleration, velocity, and position of the retinal slip, a constant, and the delay between the onset of stimulus motion and spike discharge, respectively. The delay between the retinal slip and the spikes was searched globally from 30 to $70 \mathrm{~ms}$.

\section{RES ULTS}

\section{CS and SS during OFR}

We recorded SS and CS during OFR from 34 Purkinje cells. Figure 1 shows stimulus and eye movement (velocity, acceleration, and position) and examples of the responses to upward and downward stimulus motion at $80^{\circ} / \mathrm{s}$. The characteristic short latency $(\sim 50 \mathrm{~ms}$ from stimulus to movement) and the complex acceleration of OFR were evident (Miles et al. 1986).

The Purkinje cells were categorized into two groups. One population (23/34 cells) exhibited an increase in CS and a decrease in SS firing rate in response to upward moving stimuli (Fig. 1G), and an increase in SS and a decrease in 
CS firing rate in response to downward moving stimuli (Fig. $1 H$ ). These cells were termed $\mathrm{V}$ cells. The other population (11/34 cells) exhibited an increase in SS and a decrease in CS firing rate in response to ipsilateral stimulus motion, and an increase in CS and a decrease in SS firing rate in response to contralateral stimulus motion. These cells were termed horizontal cells ( $\mathrm{H}$ cells).

\section{Directional tuning of CS (experiment 1)}

To quantify the spatial tuning characteristics of SS and $\mathrm{CS}$, the responses to moving the stimulus in eight different directions at $80 \%$ s were recorded (Fig. 2). The aggregated activities of eight V cells are shown. Both SS and CS were modulated by vertically moving stimuli, but they were not modulated by horizontal stimuli. Both SS and CS had some degree of spontaneous firing. Downward moving stimuli elicited increases in the SS firing rate and decreases in CS rate, both beginning $\sim 40 \mathrm{~ms}$ after the onset of stimulus motion. Upward moving stimuli elicited decreases in the SS firing rate and increases in CS rate with similar (40 ms) latencies. The latencies of the changes in SS and CS are more quantitatively examined later by analysis of their temporal profiles.

The preferred directions of SS and CS for individual cells are shown in Fig. 3, $A$ and $B$. Preferred directions of the SS and CS for each cell were calculated as described in METHODS. The mean of the preferred directions of SS for V cells
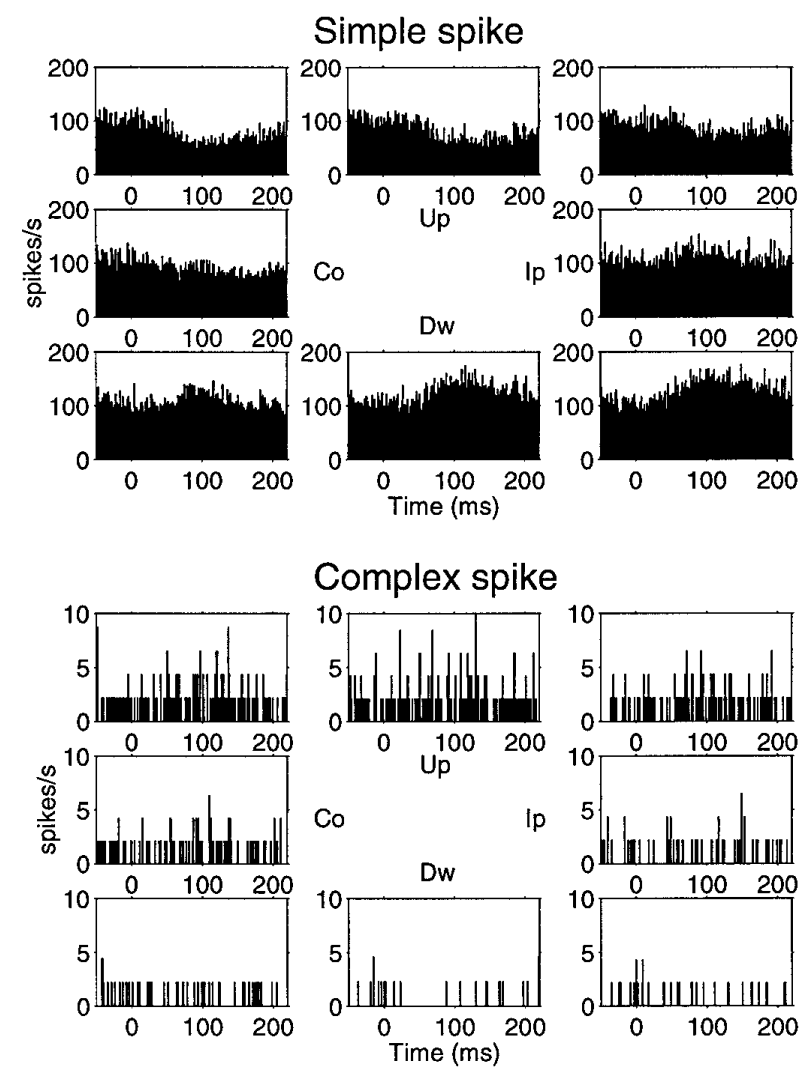

FIG. 2. SS and CS peristimulus histograms in response to moving the stimulus in 8 different directions. Aggregated responses from $8 \mathrm{~V}$ cells are shown. Position of each histogram corresponds to the direction of the stimulus motion. Ip, ipsilateral; Up, upward; Co, contralateral; Dw, downward.
A
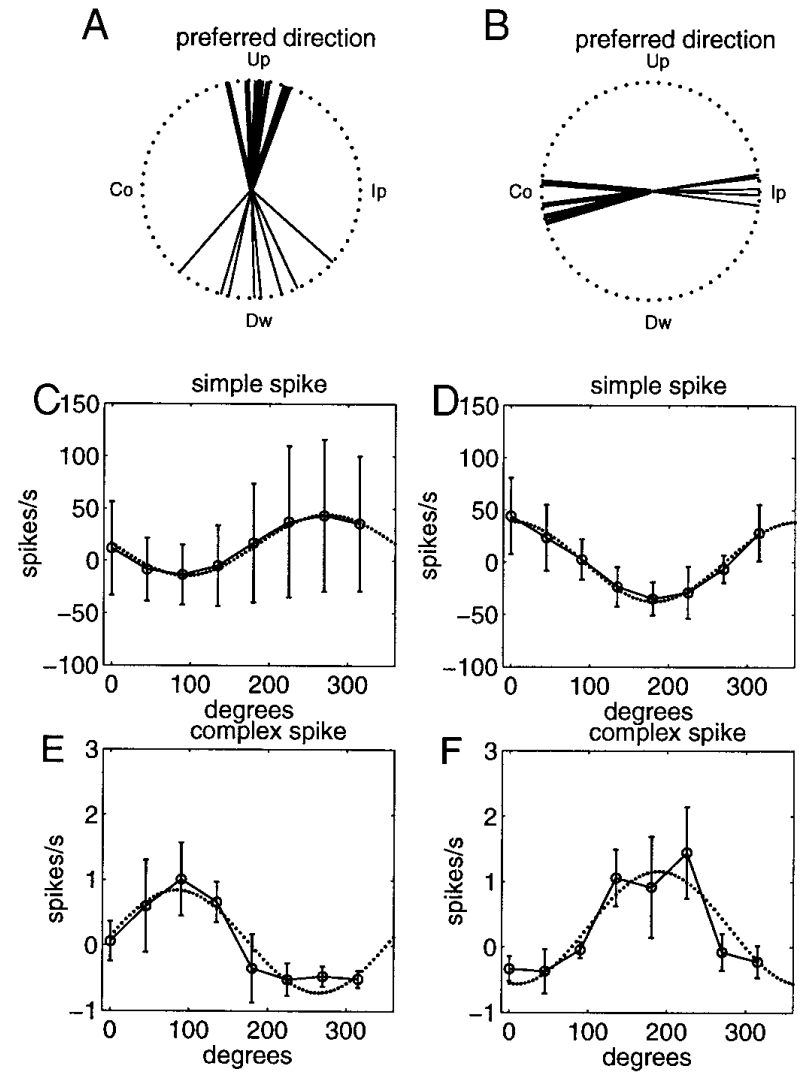

FIG. 3. Directional tuning properties of SS and CS. Preferred directions of SS (thin lines) and CS (thick lines) for $8 \mathrm{~V}$ cells $(A)$ and $5 \mathrm{H}$ cells $(B)$ are shown. $C-F$ : mean \pm SD modulation of SS and CS in V cells ( $C$ and $E$ ) and $\mathrm{H}$ cells ( $D$ and $F$ ) as a function of the direction of stimulus motion. Abscissa $(C-F)$ is the direction of stimulus motion in degrees measured counterclockwise from the ipsilateral direction (ipsilateral $=0^{\circ}$, upward $=$ $90^{\circ}$, contralateral $=180^{\circ}$, and downward $=270^{\circ}$ ). Means were fitted by a cosine tuning function (dotted line).

was $273.7 \pm 27.4^{\circ}$ (mean $\pm \mathrm{SD}$ ), that of SS for $\mathrm{H}$ cells was $1.8 \pm 6.7^{\circ}$, that of CS for V cells was $84.7 \pm 10.7^{\circ}$, $\mathrm{CS}$ for $\mathrm{H}$ cells was $189.4 \pm 5.6^{\circ}$. The mean difference between the preferred directions of SS and CS was $173 \pm 16^{\circ}$, which is close to $180^{\circ}$ (Fig. 3, $A$ and $B$ ). Thus the reciprocity between the preferred direction of SS and CS was shown.

To quantify the directional dependency of the SS and CS modulations, the directional tuning data of SS or CS averaged over the $\mathrm{V}$ cell or the $\mathrm{H}$ cell population were fitted by a cosine function of the direction of stimulus motion

$$
f=a\left[\cos \left(\theta-\theta_{p}\right)\right]+b
$$

where $f$ and $\theta$ denote the mean firing rate and the direction of stimulus movement $(\theta=0,45,90,135,180,225,270$, and $315^{\circ}$ ), respectively. The preferred directions of the averaged data $\left(\theta_{p}\right)$ were computed by averaging the preferred directions across the population. $a$ and $b$ denote the regression coefficient and the intercept of the regression equation, respectively, which were determined by the least-square method. $a$ and $b$ indicate the magnitude of direction-dependent modulation of the firing rate and the spontaneous firing rate, respectively. For the SS data, $a$ and $b$ were 29.2 and 14.8 in V cells and 38.2 and 0.7 in $\mathrm{H}$ cells. For the CS data, $a$ and $b$ were 0.78 and 0.06 in V cells and 0.86 and 0.3 in $\mathrm{H}$ cells. The averaged data and the fitted curves are shown 
in Fig. 3, $C-F$. The data and the fitted curves were well correlated ( $r=0.99$ for SS in V cells, $r=0.99$ for SS in $\mathrm{H}$ cells, $r=0.96$ for $\mathrm{CS}$ in $\mathrm{V}$ cells, and $r=0.90$ for $\mathrm{CS}$ in $\mathrm{H}$ cells), indicating that the $\mathrm{CS}$ and $\mathrm{SS}$ directional tuning characteristics were well modeled by the cosine function. The cosine directional tuning curves of SS and CS were $180^{\circ}$ out of phase. Because the mean direction-dependent modulation of the CS firing rate $(a=0.82)$ was 0.024 of that of SS $(a=33.7)$, the mean change in the CS firing rate depending on stimulus directions was only $2.4 \%$ of that of SS.

When the preferred direction of SS was plotted against that of CS as in Fig. 4, the slope of the regression line was close to $1.0(0.82)$ and its intercept was close to $180^{\circ}\left(155^{\circ}\right)$. These results provide quantitative evidence that the spatial tuning properties (including the preferred direction) of CS are opposite to those of SS. In 13 cells examined, the preferred direction of SS recorded from each cell correlated with the preferred direction of CS recorded from the same cell with a coefficient of $0.90(P=0.001)$. The data were separated into two clusters (i.e., $\mathrm{H}$ and $\mathrm{V}$ cells). Although no significant correlation existed when each cluster of data were regressed separately, the above significant correlation for all the data at least indicates global reciprocity of the preferred directions of the SS and CS.

\section{Effects of stimulus velocity on CS and SS (experiment 2)}

Twelve cells were studied and findings for one example are shown Fig. 5. The SS firing rate increased and the CS rate decreased with increased downward stimulus velocity (retinal slip velocity) and the resulting downward eye movement. Moreover, the SS firing rate decreased and the CS rate increased with increased upward stimulus velocity and the resulting upward eye movement.

To quantify the correlation between the SS and CS firing rates and retinal slip or eye velocities, the mean SS and CS firing rates were plotted against mean retinal slip and mean eye velocity. Considering its time delay, eye movement was averaged over the time interval from 50 to $300 \mathrm{~ms}$ after the onset of stimulus motion. SS and CS were averaged from 40 to $290 \mathrm{~ms}$ after the onset of stimulus motion. Retinal slip was averaged from 0 to $250 \mathrm{~ms}$ after the onset of stimulus motion. The mean SS firing rate was a monotonically de-

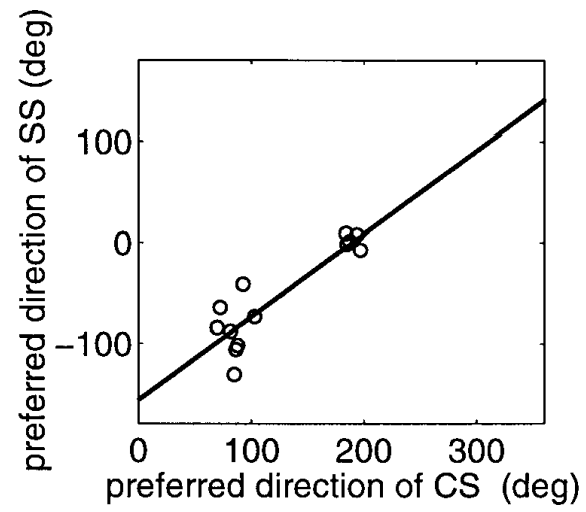

FIG. 4. Preferred direction of SS for each cell is plotted against that of CS. Line represents the linear regression of the data.

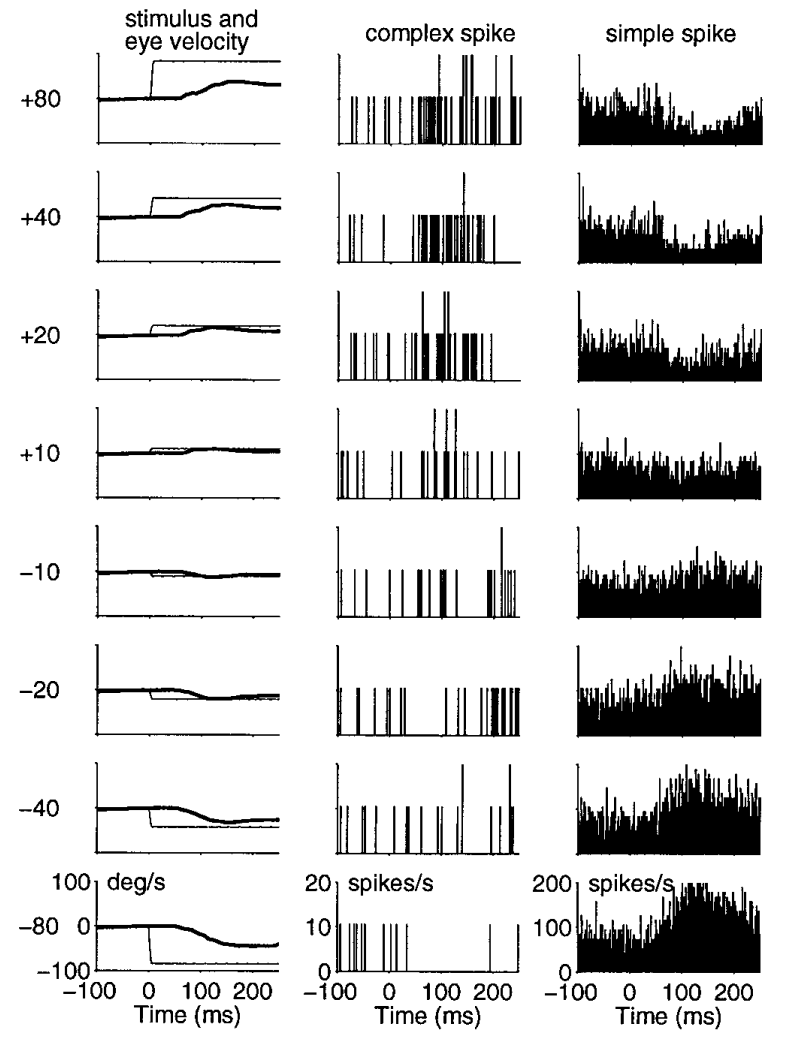

FIG. 5. SS and CS of an example $\mathrm{V}$ cell in response to a wide range of stimulus velocities. Stimulus was moved vertically at $+80,+40,+20$, $+10,-10,-20,-40$, and $-80 \%$ s. Upward motion was assigned positive polarity. Mean stimulus (thin line) and eye (thick line) velocities (left) and peristimulus time histograms with CS (middle) and SS (right) binned in 1-ms intervals are shown for each stimulus velocity for which 95 trials were obtained.

creasing function of the slip velocity. The curve from each individual cell was a sigmoid function, that is, a decreasing, monotonic, saturating function (Fig. 6A). In contrast, the mean SS firing rate was approximately a linear function of the eye velocity (Fig. 6B). The correlation coefficient between the mean SS firing rate and eye velocity for each of 12 cells was calculated and then averaged ( mean $=-0.99)$. The absolute value of the mean correlation coefficient $(-0.91)$ between the SS firing rates and the slip velocities was statistically significantly smaller $(P=0.0001)$ than that between SS and eye velocity. This observation provides quantitative evidence that the relationship between the SS firing rate and eye velocity is more linear than the relationship between the SS firing rate and slip velocity.

The mean CS firing rate of each cell was approximately an increasing, saturating function of the slip velocity, although some exceptions can be seen especially at large slip velocities (Fig. 6C). At stimulus velocities between 40 and $80^{\circ} \mathrm{s}$, some cells exhibited an increase, some a decrease, and some no change in CS firing rate. As found for SS, the relationship between the mean $C S$ firing rate and the eye velocity was more linear (Fig. 6D). The mean correlation coefficient between CS firing rate and eye velocity (0.93) was statistically larger than $(P=0.008)$ that between CS firing rate and slip velocity $(0.89)$, again providing quantitative evidence that the relationship between CS firing rate 
A
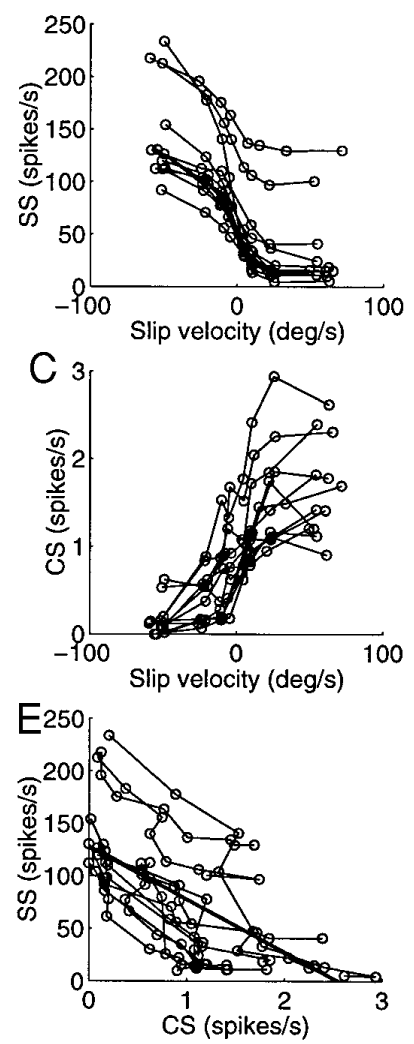

$\mathrm{B}$
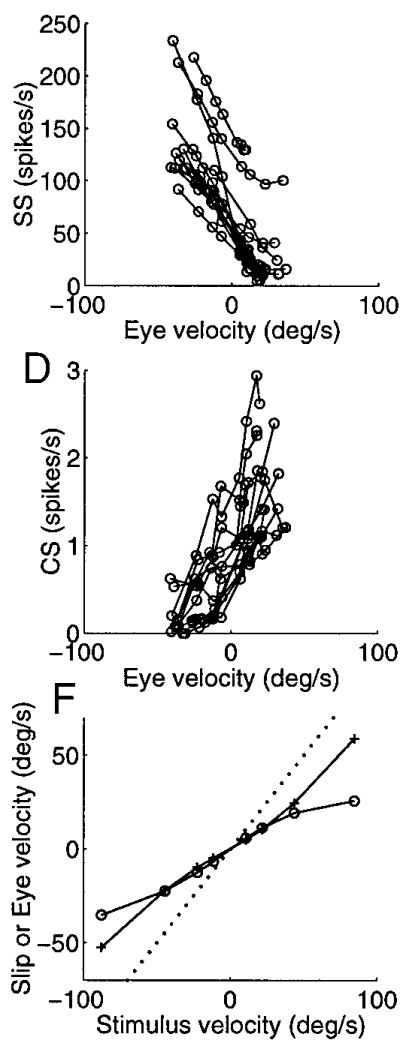

FIG. 6. Velocity tuning curves of SS and CS. Following relations are shown: SS firing rate and retinal slip velocity $(A)$, SS firing rate and eye velocity $(B)$, CS firing rate and retinal slip velocity $(C)$, CS firing rate and eye velocity $(D)$, SS and CS firing rate $(E)$. Bold solid line shows the regression line of all the data. $F$ : retinal slip velocity (circle) as a function of the stimulus velocity, and the eye velocity (cross) as a function of the stimulus velocity. Dotted line shows the line with a slope of 1.0 and passing through the origin. Retinal slip was calculated by subtraction of eye movement from stimulus movement. Upward or contralateral motion was assigned positive polarity. Each set of data points connected by a line represents data from an individual cell $(n=12)$.

and eye velocity is more linear than the relationship between CS firing rate and slip velocity.

The correlation coefficient between the mean SS and CS firing rate at each stimulus velocity was calculated for each of 12 cells (Fig. $6 E$ ) and then averaged (mean $=-0.91 \pm$ $0.06 ; P=0.05)$. This result demonstrates that there is a reciprocal relationship between the mean SS firing rate and the mean CS firing rate with respect to their stimulus and eye velocity dependence. The very large negative slope $(-48.2)$ of the regression line in Fig. $6 E$ for the average data of 12 cells indicates that modulation of CS firing rate depending on different stimulus speeds was opposite in sign and only $2.1 \%$ of that of SS.

SS and CS dependencies on either the slip velocity or the eye velocity were examined in Fig. $6, A-D$. Because OFR is essentially induced by the retinal slip, one may wonder that the slip velocity is very highly correlated with the resultant eye velocity, thus the above analyses might not be sensible. Plotting the averaged slip velocity (cross) and the averaged eye velocity (circle) as functions of the stimulus velocity resolved this issue (Fig. $6 F$ ). Even in the small stimulus

velocity range (from -40 to $40^{\circ} / \mathrm{s}$ ), the two curves had opposite curvatures. Furthermore, for the largest stimulus speeds $(-80$ and $80 \%$ s $)$, the eye velocity showed clear signs of saturating, whereas the retinal slip kept increasing. Thus even for the averaged behavior, the retinal slip and the eye movement were considerably different. Marked differences in their transient behaviors will be given in the following text.

\section{Temporal patterns of CS firing rates (experiment 3)}

In V cells $(n=9)$, the SS firing rate decreased and the CS firing rate increased in response to upward $80^{\circ} / \mathrm{s}$ stimulus motion (Fig. 7A). Moreover, CS and SS of an individual cell appeared to be affected to similar extents by the same stimulus motion (e.g., cells 1 and 2 exhibited relatively small changes in both CS and SS firing rate, whereas cell 3 exhibited relatively large changes in both CS and SS firing rate). Furthermore, although the percent change in the SS firing rate was much larger than that of CS (note the 10 times difference in ordinate scales between the left and right columns), the SS temporal firing profiles were similar to those of CS if the sign was reversed and the magnitude scaled.

There was a significant cell-to-cell negative correlation $(-0.76, P=0.01)$ between the mean magnitude of change from the spontaneous activity in the SS and CS firing rates

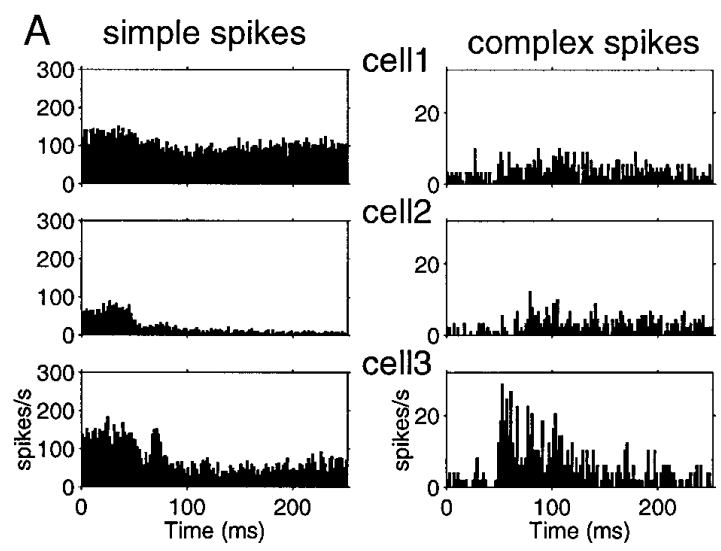

B

C
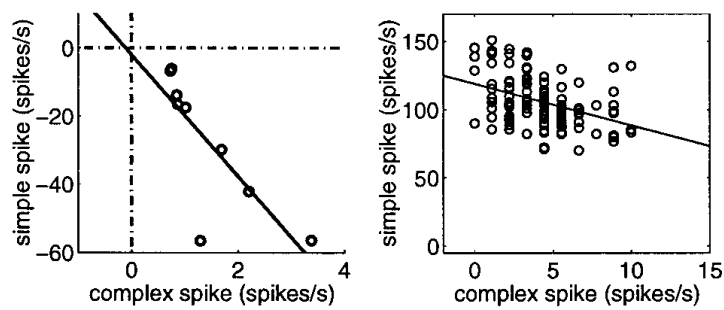

FIG. 7. Reciprocal relationships between the CS and SS firing rates. $A$ SS and CS firing rates evoked by upward stimulus motion at $80^{\circ} / \mathrm{s}$ (1-ms bins from 0 to $300 \mathrm{~ms}$ after the onset of stimulus motion) for 3 example cells. $B$ : mean percent change in SS firing rate for each cell is plotted against that of CS. Solid line represents the linear regression of the data. $C$ : instantaneous SS firing rates (binned in 2-ms intervals from 0 to 300 ms after the onset of stimulus motion) of cell 1 shown in $A$ were plotted as a function of those for CS in the same time bins. Solid line represents the linear regression of the data $(n=150 ; r=-0.38 ; P=0.0001)$. 


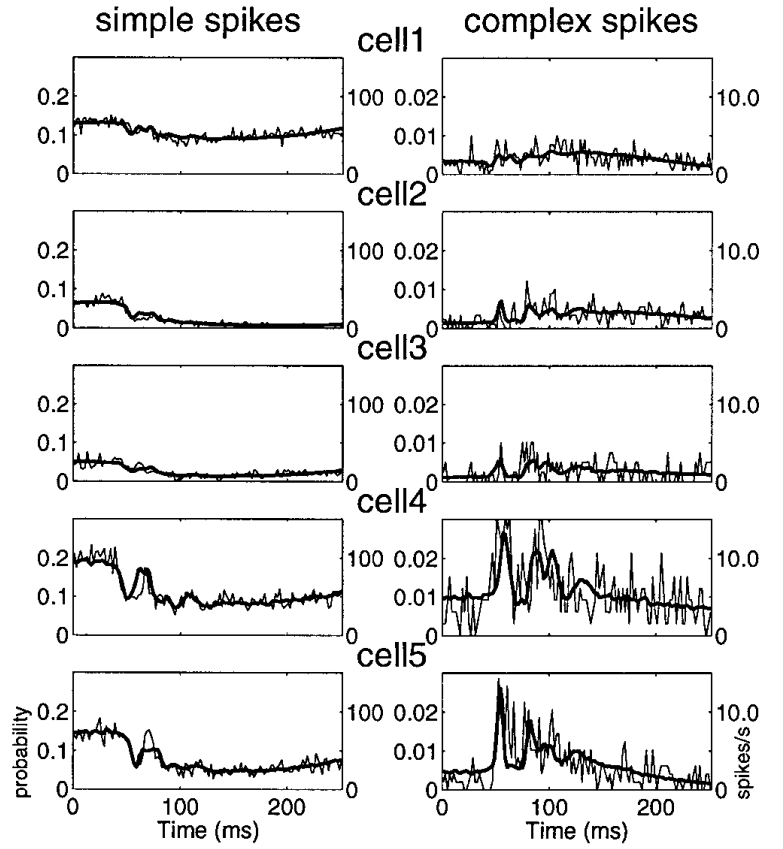

FIG. 8. Reproduction of the SS (left) and CS (right) firing probability from the eye movement for 5 example $\mathrm{V}$ cells in response to upward stimulus motion at $80^{\circ} / \mathrm{s}$ using a generalized linear model. Thin curves show the observed firing rate in 2-ms bins, and the thick curves show the estimated firing probability within the corresponding time bin. Note that the ordinate scales for the firing rate (right) and the firing probability (left) were matched such that the asymptote of the former overlaid that of the latter. Accumulated trial numbers for the 5 cells were 901, 899, 396, 327 , and 487 from top to bottom. Data are shown from top to bottom in order of increasing mean change in firing rate in response to stimulus motion.

in response to upward $80^{\circ} / \mathrm{s}$ stimulus motion (Fig. $7 \mathrm{~B}$ ). This result statistically supports the above qualitative observation suggesting that the magnitude of change in the CS firing rate parallels the magnitude of change in the SS firing rate. Because the slope of the regression line is -17.9 , the population mean change in CS firing rate is $5.6 \%$ of that of SS, for the preferred direction of CS and the anti-preferred direction of SS. The $y$-axis intercept of the regression line (Fig. $7 B$ ) was not significantly different from zero $(P=0.23)$, which suggests that the SS firing rate is unmodulated if the CS firing rate is unmodulated and vice versa.

There was a statistically significant negative correlation $(P=0.0001)$ between the instantaneous CS and SS firing rates within the same 2-ms time bin (Fig. 7C) for cell 1 shown in Fig. 7A. The instantaneous $\mathrm{SS}$ and $\mathrm{CS}$ firing rates were negatively correlated for all nine cells examined in experiment 3 . For seven of the nine cells, the negative correlation was statistically significant $(P<0.01)$. This analysis provides statistical evidence that the SS temporal firing profile is similar but of opposite sign to the CS temporal firing profile.

\section{Reproduction of firing probability of CS}

The result of using a generalized linear model of eye movement to reproduce the SS and CS firing probability in experiment 3 is shown in Fig. 8 for five individual cells. The mean correlation coefficient between the observed firing rate and the estimated firing probability was $0.84 \pm 0.12$ for
$\mathrm{SS}$ and $0.48 \pm 0.10$ for CS for the nine cells in experiment 3. The mean deviance of the CS firing probability for the eye movement model (equation 2) was smaller than that of the SS (Table 1), indicating that the CS firing probability was more accurately reproduced than that of the SS. Furthermore, the deviance of the CS firing probability was smaller than that of the SS firing probability on an individual cell basis for seven of the nine Purkinje cells analyzed. As the number of trials performed while recording from a single cell approaches infinity, the firing rate (spikes/s) multiplied by the time bin ( $s$ ) should approach the firing probability $p$. On the other hand, if the trial number $n$ is finite, there are large fluctuation in firing rate with a standard deviation $\sqrt{n p(1-p)}$ due to the binomial distribution (see METHODS). Thus the rapid fluctuation of the firing rate from the predicted firing probability observed in Fig. 8 is not mainly an error due to the model but rather sampling noise inherent to the stochastic spike-count data itself.

A generalized linear model of eye movement also was used to reproduce the SS and CS firing probability from experiment 2. A single set of parameters was estimated for each cell to reproduce the firing patterns for all stimulus velocities. As in experiment 3, the mean deviance of the CS firing rate was smaller than that of the SS (Table 1) and the deviance of the CS firing rate was smaller than that of the SS firing rate for all 12 individual cells recorded in experiment 2. Thus these data also indicate that the CS firing probability was better reproduced from eye movement than was the SS firing probability.

We examined whether temporal patterns of firing probability in SS and CS encode significantly sensory or motor information. The firing probabilities of CS and SS were reconstructed via the generalized linear model of eye movement (Eq. 2) or retinal slip ( $E q .6$ ), then the deviances were compared between the eye movement model and the retinal slip model. To improve the data reliability, we averaged the data from nine cells recorded in experiment 3 as shown in Fig. 9. Because of this population averaging, the stochastic noise in the firing rate of CS was reduced. The temporal patterns of retinal slip position, velocity, and acceleration and eye position, velocity, and acceleration and SS and CS elicited by upward $80^{\circ} / \mathrm{s}$ stimuli are shown in Fig. 9. Thick lines in Fig. 9, $G$ and $I$, show the firing probability reconstructed for SS and CS respectively by the generalized linear model of the retinal slip. Thick lines in Fig. 9, $H$ and $J$,

TABLE 1. Mean deviances in estimations of firing probability from eye movement and retinal slip

\begin{tabular}{lccccc}
\hline \hline & \multicolumn{2}{c}{ Upward $80 \%$ Stimulus } & & \multicolumn{2}{c}{ 8-Speed Stimuli } \\
\cline { 2 - 3 } \cline { 5 - 6 } & Eye Movement & Retinal Slip & & Eye Movement & Retinal Slip \\
\hline SS & $191.3 \pm 88.0$ & $171.6 \pm 53.9$ & & $358.5 \pm 150.5$ & $423.4 \pm 183.4$ \\
CS & $150.9 \pm 24.1$ & $138.5 \pm 11.4$ & & $78.5 \pm 11.3$ & $83.0 \pm 12.9$ \\
\hline
\end{tabular}

Comparison of mean deviances $( \pm$ SD) in estimations of firing probability from eye movement and retinal slip. The two left columns show the results from experiment 3 , which used $80 \%$ s stimulus in the preferred direction for complex spikes (CS), and the right two columns show the results from experiment 2, which used 8 or 6 speed stimuli in both the preferred and anti-preferred directions for CS and simple spikes (SS). 

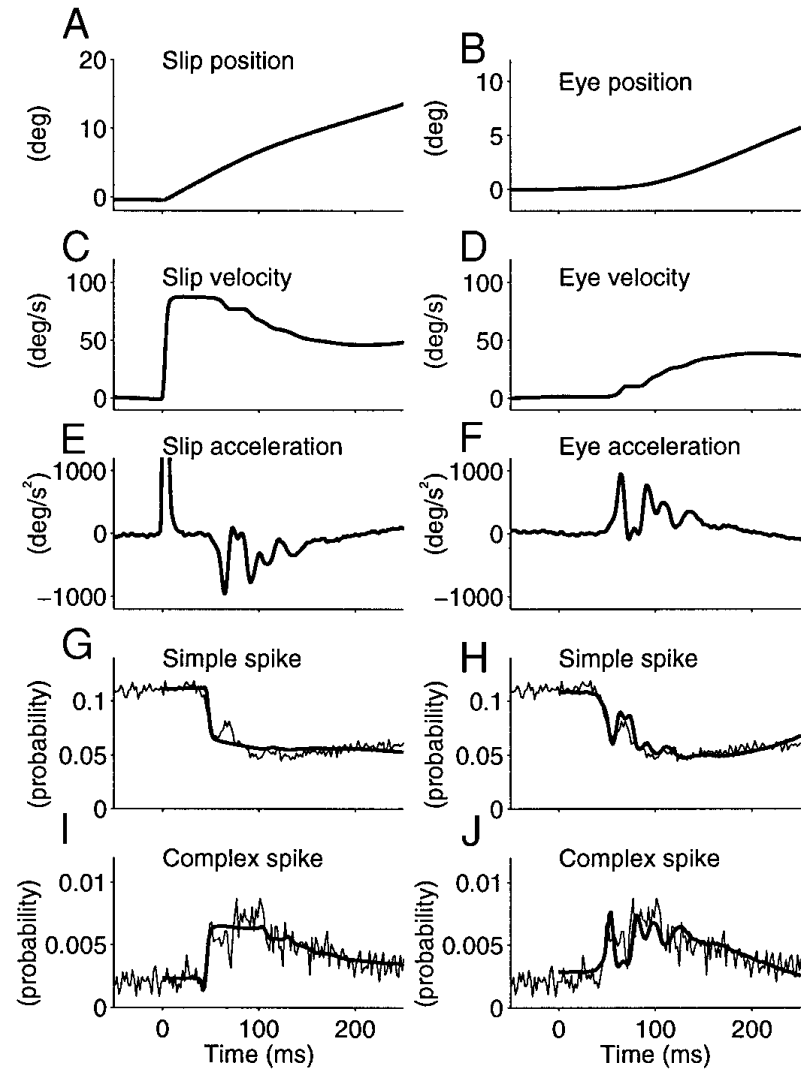

FIG. 9. Population average of the retinal slip, eye movement, CS and SS of 9 cells in experiment 3 (5,214 trials). Left : retinal slip position $(A)$, velocity $(C)$, and acceleration $(E)$ in responses to $80^{\circ} \%$ s upward stimulus motion. Right: eye position $(B)$, velocity $(D)$, and acceleration $(F)$ responses to the same stimulus motion. $G$ and $H$ : thin traces show the population average of firing probability of SS. Thick traces in $G$ and $H$ show the estimated firing probability of SS from retinal slip and eye movement, respectively. $I$ and $J$ : thin traces show the population average of firing probability of CS. Thick traces in $I$ and $J$ show the estimated firing probability of CS from retinal slip and eye movement, respectively.

show the firing probability reconstructed by the generalized linear model of eye movement for SS and CS, respectively.

It may appear superficially that the temporal patterns of the retinal slip and the eye movement are similar and thus that it is statistically difficult to discriminate which signal better reconstructs the firing frequency patterns. But actually even the position temporal patterns are quite different between the two signals unless an appropriate time shift is introduced, whereas the velocity and acceleration are entirely different with negative correlations. The firing data were best modeled by retinal slip $\sim 40 \mathrm{~ms}$ time delayed, and it was modeled best by the eye movement $\sim 10 \mathrm{~ms}$ time advanced. Thus for estimating statistically the extent of similarity of the two signals, we first time delayed the retinal slip by $40 \mathrm{~ms}$ and time advanced the eye movement by $10 \mathrm{~ms}$ and then calculated the correlation of the two signals. The correlation coefficient between retinal slip acceleration 0-200 ms after onset of the stimulus motion and eye acceleration 50-250 ms from onset of the stimulus motion was -0.012 . Thus the acceleration patterns were little correlated. The correlation coefficient between velocity of retinal slip and velocity of eye movement was -0.60 . Thus the velocity patterns were negatively correlated. The coefficient for position was 0.97 . Thus in summary, the position patterns were highly positively correlated, but the dynamic components (acceleration and velocity) of the two temporal patterns were entirely different.

Comparison of the deviances for the eye movement model and the retinal slip model, as shown in the left two columns of the Table 1 indicates that the SS and CS firing probabilities were reproduced as well or better from the retinal slip than from eye movement for the upward $80^{\circ} / \mathrm{s}$ stimulus in experiment 3. But we do not think this is a statistically important observation. To reliably and more rigorously compare the two statistical models in reproducing the experimental data, the generalization capability of the models should be tested using the data from experiment 2 because a large variety of stimuli and responses are essential to test the goodness of the models. The mean deviances in experiment 2 are shown in the right two columns of Table 1. Here, both the SS and CS were better reproduced from the eye movement than the retinal slip. Instead of directly comparing the deviance values themselves, an index of the sensory-motor nature of the signals was calculated as the deviance for the eye movement divided by that for the retinal slip. If the index is smaller than 1 , the signal will be more motor in nature, whereas if it is larger than 1 , the signal will be more sensory. First, because the mean of the index for CS (0.95) was close to 1 , CS were equally well reproduced from either the retinal slip or the eye movement. Second, the mean of the index for SS (0.85) was smaller than that for CS, suggesting that SS are more motor in nature than CS (and conversely, that CS are more sensory in nature than SS).

The generalized linear model of the eye movement shown in Eq. 2 nonlinearly transforms the linear weighted summation of the eye acceleration, eye velocity, eye position, and the constant term, $M \cdot \ddot{\theta}(t+\delta)+B \cdot \dot{\theta}(t+\delta)+K \cdot \theta(t+$ $\delta)+C$ by the sigmoid function $S$ defined in $E q$. 3. The bold solid curves in Fig. 10, $A$ and $B$, denote this summation, that is, the argument of $S$ or the contents of the square bracket in $E q .2$ for the SS and CS, respectively. Here, we use the same population data from the 9 cells in experiment 3 , which were already shown in Fig. 9, $B, D, F, H$, and $J$. The noisy curves denote the inverse of the sigmoid function of the actual firing data: $S^{-1}\left(y_{i} / m\right)=\log \left[\left(y_{i} / m\right) /\left(1-y_{i} / m\right)\right]$. Because the bold solid curve $S^{-1}(p)$ well approximates this noisy curve, the good fit of the generalized linear model was reconfirmed. The four thin solid curves shown in Fig. 10 indicate the four terms, i.e., $M \cdot \ddot{\theta}(t+\delta), B \cdot \dot{\theta}(t+\delta), K \cdot \theta(t$ $+\delta$ ), and $C$ for the SS and CS, in $A$ and $B$, respectively. $C$ of the CS was smaller than that of the SS by $\sim 4$, indicating that the spontaneous firing rate of the CS was about exp $(-4)$ $=0.02$ times of that of the SS. The acceleration, velocity and position curves of the CS shown in Fig. $10 \mathrm{~B}$ had the opposite polarity but similar magnitudes to those of SS in Fig 10A. This reconfirmed that the temporal patterns of firing frequency for the CS are similar to those for the SS if the sign is reversed and the magnitude is scaled by dividing by $\exp (-4)=0.02$.

Next, we examined the relative contributions of the three factors (acceleration, velocity, and position) in reconstructing the SS and CS temporal profiles by calculating the following variance accounted for (VAF) of the eye acceleration, velocity, and position, respectively 
A

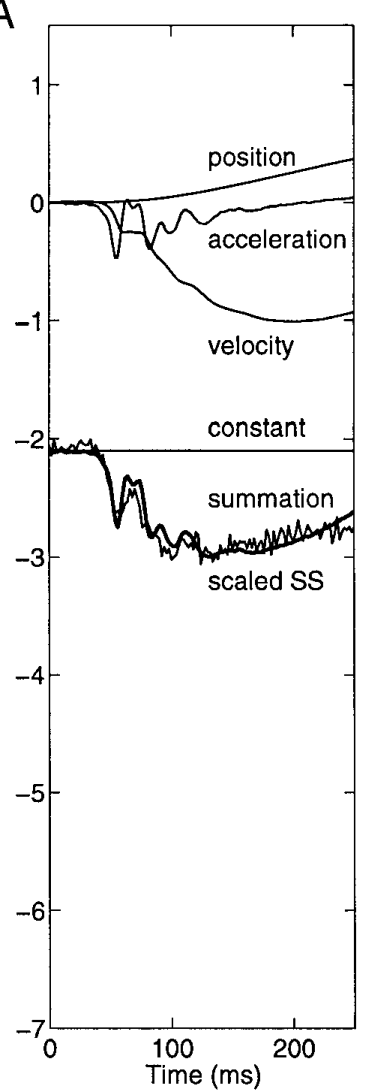

$\mathrm{B}$

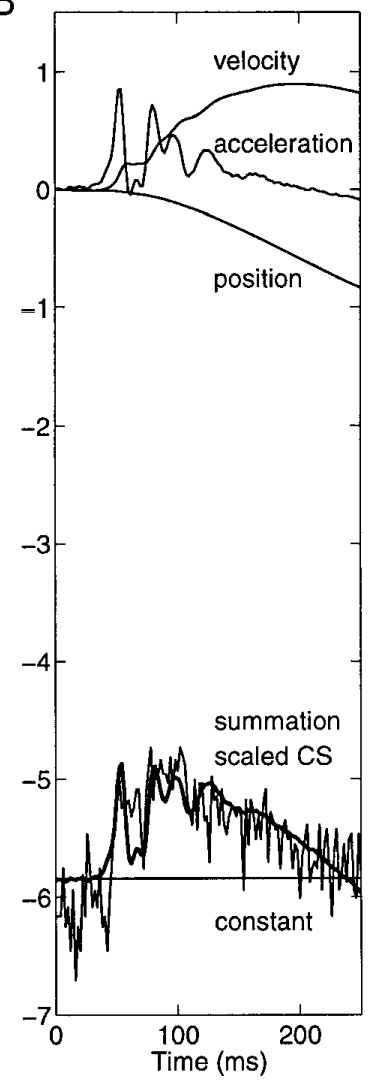

FIG. 10. Bold solid curves in $A$ and $B$ indicate the linear weighted summation of the eye acceleration, eye velocity, eye position and the constant term, $M \cdot \ddot{\theta}(t+\delta)+B \cdot \theta \cdot(t+\delta)+K \cdot \theta(t+\delta)+C$ for the SS and CS, respectively. Four thin solid curves shown in $A$ for the SS and in $B$ for the CS indicate the 4 terms in these summations. Noisy curves indicate the inverse of the sigmoid function of the actual firing rates. We use the same aggregated data from the 9 cells in experiment 3 , which were already shown in Fig. 9, $B, D, F, H$, and $J$.

$$
\begin{aligned}
& \operatorname{VAF}_{\mathrm{a}}=1-\frac{V\left\{S^{-1}[p(t)]-M \cdot \ddot{\theta}(t+\delta)\right\}}{V\left[S^{-1}[p(t)]\right]} \\
& \mathrm{VAF}_{\mathrm{v}}=1-\frac{V\left\{S^{-1}[p(t)]-B \cdot \dot{\theta}(t+\delta)\right\}}{V\left\{S^{-1}[(p(t)]\}\right.} \\
& \mathrm{VAF}_{\mathrm{P}}=1-\frac{V\left\{S^{-1}[p(t)]-K \cdot \theta(t+\delta)\right\}}{V\left\{S^{-1}[p(t)]\right\}}
\end{aligned}
$$

The VAF indicates what proportion of the total modulation in the $S^{-1}$ transformed firing frequency could be accounted for by each of the three terms. $V[x]$ denotes the variance of $x$. A larger VAF indicates a larger contribution of that component. $\mathrm{VAF}_{\mathrm{a}}, \mathrm{VAF}_{\mathrm{v}}$, and $\mathrm{VAF}_{\mathrm{p}}$ for the SS were 0.05, 0.51 , and -0.55 , respectively. $\mathrm{VAF}_{\mathrm{a}}, \mathrm{VAF}_{\mathrm{v}}$, and $\mathrm{VAF}_{\mathrm{p}}$ for the CS were $0.34,-0.24$, and -0.29 , respectively. These results indicate that the eye velocity component was the most dominant in the SS and eye acceleration component was the most dominant in the CS.

The ratio of the velocity and acceleration coefficients $(B$ ) $M)$ in the generalized linear model of eye movement ( $E q$. 2 ) for the population data shown in Figs. 9 and 10 was computed. $(B / M)$ for SS was 55 and for CS was 26 . This confirms that the SS contained the larger velocity component or the CS contained the larger acceleration component.

In both experiment 3 and experiment 2, the mean acceleration, velocity, and position coefficients of eye movement $(M, B$, and $K)$ for CS and SS generally had opposite signs but were of the same order of magnitude (Table 2). The mean $C$ of $\mathrm{CS}$ was smaller than that of SS by $\sim 4$. This also indicates a lower firing probability of the CS than the SS. Taken together, this indicates that the percent change in the CS firing rate is approximately $\exp (-4)=0.02$ that of the $\mathrm{SS}$ firing rate. The onset of SS and CS modulation preceded the onset of eye movement by a similar amount: the average $\delta(E q .2)$ from 21 cells from experiment 2 and experiment 3 was $5.1 \pm 10.3 \mathrm{~ms}$ for $\mathrm{CS}$ and $10.8 \pm 6.0 \mathrm{~ms}$ for SS (means $\pm \mathrm{SD}$; not significantly different $P>0.05$; see also Table 2). Because $M, B, K, C$, and $\delta$ reflect the temporal firing probability profile, these results indicate that the CS temporal firing probability profile was similar to that of SS if the sign was reversed and the modulation amplitude scaled. Thus these data provide additional quantitative evidence with high temporal resolution (2-ms bin) that the SS and CS temporal profiles were similar but opposite in sign.

We have demonstrated previously that SS recorded in the VPFL exhibit temporal firing profiles that closely follow an inverse dynamics representation of eye movements and that the ratio between the acceleration and velocity coefficients is close to that of motor neurons, thus indicating their role as the dynamic motor commands (Gomi et al. 1998; Kawano et al. 1996; Shidara et al. 1993). In the present study, we modeled firing probability by generalized linear models instead of using linear models for firing frequency. Because the sigmoid (logarithmic) function in the generalized linear model can be approximated by an exponential function if its argument is negative and its absolute value is large, the acceleration, velocity, and position coefficients of the linear model can be approximated from the corresponding coefficients of the generalized linear model by multiplying by $\exp (C)$. Thus the ratio of the coefficients can be compared directly between the linear model and the generalized linear model used in the present study.

The mean velocity and acceleration coefficient ratio of eye movement model $(B / M)$ for 21 cells in experiments 2 and 3 for SS was 56, which is close to that for motor neurons (67) (Keller 1973); thus these results are consistent with the hypothesis that SS provide dynamic motor commands (Gomi et al. 1998; Kawano et al. 1996; Shidara et al. 1993). The mean ratio $(B / M)$ for 21 cells in experiments 2 and 3 for CS was 28. This indicated the temporal profile of SS contained the larger velocity component or that of CS contained the larger acceleration component. The same conclusion was drawn already from application of the same model to the accumulated data from nine cells in experiment 3 (Figs. 9 and 10). The VAF analysis of the same data also reconfirmed this.

Plots of the best-fit parameters of the coefficients $M, B$, $K$, and $C$ for the SS data against the best-fit parameters for the CS data for each of the 21 Purkinje cells from experiments 2 and 3 indicate that the acceleration, velocity, and position coefficients for the SS and CS data generally had opposite signs (note the quadrant) but were of the same order of magnitude, even on a cell-to-cell basis (Fig. 11). The regression lines in Fig. 11, $A$ and $B$, were constrained to pass through the origins for the following two reasons. 
TABLE 2. Estimated model parameters in the generalized linear model of eye movement

\begin{tabular}{|c|c|c|c|c|}
\hline & \multicolumn{2}{|c|}{ Upward $80 \%$ s Stimulus } & \multicolumn{2}{|c|}{ 8-Speed Stimuli } \\
\hline$B$ & $-0.036 \pm 0.020$ & $0.023 \pm 0.017$ & $-0.036 \pm 0.010$ & $0.049 \pm 0.039$ \\
\hline$K$ & $0.10 \pm 0.096$ & $-0.14 \pm 0.11$ & $0.078 \pm 0.0071$ & $-0.22 \pm 0.34$ \\
\hline$C$ & $-2.2 \pm 0.62$ & $-5.9 \pm 0.67$ & $-2.4 \pm 0.62$ & $-6.5 \pm 1.1$ \\
\hline$\delta, \mathrm{ms}$ & $10.4 \pm 4.9$ & $6.2 \pm 10.2$ & $11.0 \pm 6.9$ & $4.2 \pm 10.7$ \\
\hline
\end{tabular}

Comparison of estimated model parameters in the generalized linear model of eye movement described in $E q .2$ (means \pm SD) for reconstruction of SS and CS firing probabilities. The left two columns show the results from experiment 3, and the right two columns show the results from experiment 2 .

First, the results shown in Fig. $7 B$ indicate that SS were unmodulated if CS were unmodulated. This suggests that the inverse dynamics coefficients were zero for both SS and $\mathrm{CS}$, so that the origin was a default data point. Second, the results of experiment 1 indicate that both CS and SS were unmodulated by the stimulus direction perpendicular to their optimal and antioptimal directions. Thus a large number of data points concentrate on the origin. The slopes of the regression lines for $M(P=0.02)$ and $B(P=0.00001)$ were significantly more negative than zero based on a Student's $t$ test. This result indicates that there are cell-to-cell negative correlations between the SS and CS coefficients and consequently cell-to-cell negative correlations between the SS and CS temporal firing patterns. $M$ and $B$ are functionally more important than $K$ because SS provide only the dynamic part of the motor commands (Gomi et al. 1998; Kawano et al. 1996; Shidara et al. 1993).
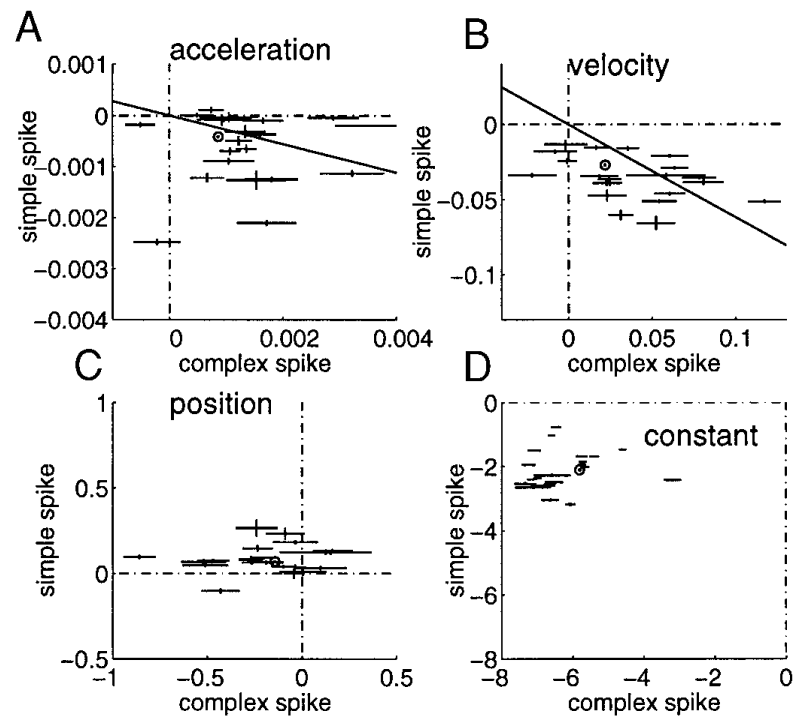

FIG. 11. Estimated parameters $M, B, K$, and $C$, and their confidence intervals are shown in $A-D$, respectively. Estimated coefficient for SS of each cell is plotted against that of CS using the same scale for the ordinate and the abscissa. Center of each cross indicates the maximum likelihood estimation, whereas the lengths of the vertical and horizontal bars indicate the square roots of the asymptotic variance for SS and CS, respectively. Crosses represent data from 9 cells responding to upward stimulus motion at $80^{\circ} \mathrm{s}$ and data from 12 cells in which 6 or 8 different velocities were used. Estimated coefficients from the averaged data to upward stimulus motion at $80^{\circ}$ /s (5,214 trials) are plotted as double circles.

\section{Short-term modulation of SS by CS}

The cross-correlation analysis was applied to the SS (Fig. $12 A$ ) and the CS (Fig. 12B) firing for nine cells presented with upward $80^{\circ} / \mathrm{s}$ stimulus motion (experiment 3 ). Figure 12 shows the results for one example cell. The apparent cross-correlation $R_{\text {app }}$ was calculated directly by CS spike trigger averaging of the SS (Fig. $12 C$ ). The stimulus-dependent cross-correlation $R_{\text {stm }}$ was similarly calculated after shuffling the impulse trains (Perkel et al. 1967; Toyama et al. 1981) (Fig. 12D). The true interaction (net crosscorrelation $R_{\text {net }}=R_{\text {app }}-R_{\text {stm }}$ ) between SS and CS (Fig. $12 E$ ) was determined by subtracting the stimulus-dependent cross-correlation from the apparent cross-correlation. The proportion of the SS discharge modulation $\mathrm{SS}_{\mathrm{CS}}$ that is a

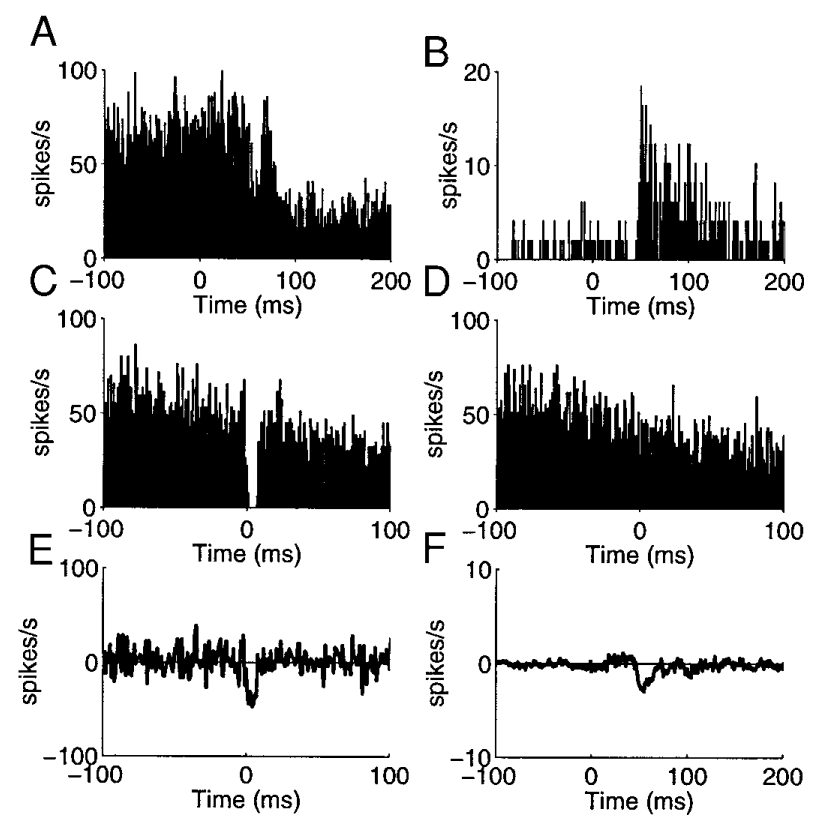

FIG. 12. Cross-correlation analysis of SS and CS. A: SS peristimulus time histogram. $B$ : CS peristimulus time histogram. $C$ : apparent crosscorrelation between SS and CS, $R_{\text {app }}$, which was directly calculated by CS spike trigger averaging of SS. $D$ : stimulus-dependent pseudo crosscorrelation between SS and CS, $R_{\mathrm{stm}}$, which was similarly calculated after shuffling the impulse trains (Perkel et al. 1967; Toyama et al. 1981). E: net cross-correlation between SS and CS, $R_{\text {net }}$, determined by subtracting the stimulus-dependent cross-correlation in $D$ from the apparent cross-correlation in $C . F$ : SS modulation, $S S_{\mathrm{CS}}$, which is accounted for by the shortterm effect on SS by CS. Data shown in $A-F$ were obtained from responses of 1 cell to upward stimulus motion presented 487 times at $80^{\circ} / \mathrm{s}$. 
direct consequence of short-term effect of CS can be evaluated by the convolution integral of the CS firing pattern with the net cross-correlation obtained above (Fig. 12F)

$$
\mathrm{SS}_{\mathrm{CS}}(t)=\int_{0}^{\infty} R_{\text {net }}(s) \mathrm{CS}(t-s) \mathrm{d} s
$$

Comparison of the convolution integral of CS (Fig. 12F) and the SS peristimulus time histogram (Fig. 12A) indicates that the estimated CS-induced SS modulation was negligible compared with the actual SS modulation; the ratio of the estimated CS-induced SS modulation (averaged over the interval from 0 to $250 \mathrm{~ms}$ from the onset of the stimulus motion after subtraction of the prestimulus firing rate) and the actual SS modulation after subtraction of the prestimulus firing rate was very small $(0.006)$ (for the 9 cells, mean $\pm \mathrm{SD}=1.2 \times$ $\left.10^{-2} \pm 1.4 \times 10^{-2}\right)$. It is important not to overestimate the stimulus-dependent pseudo correlation and, consequently, to underestimate the net correlation and the CS-induced SS modulation. The magnitude of the net cross-correlation between SS and CS observed in the present study was approximately the same order of magnitude as in previous observations (3050 spikes/s firing rate during the pause and 10-30 ms pause duration) (Sato et al. 1992; Stone and Lisberger 1990b). Consequently, the reciprocal relationship between SS and CS cannot be explained by a short-term CS-induced SS modulation. A similar conclusion was derived from studies of other species (Simpson et al. 1996).

\section{DISCUSSION}

\section{Theories of CS function}

The view that climbing-fiber inputs function as detectors of control errors is accepted rather widely but there is still much argument as to whether they act in real-time via the direct influence of CS on targets of the Purkinje cells and/ or via a short-term modulatory action on SS patterns or in longer term by inducing long-lasting changes in the potency of parallel fiber-Purkinje cell synapses. More specifically, the primary theories regarding the function of CS are summarized as follows. Some previous researchers have not excluded the possibility that multiple theories may be correct so that the CS have multiple functions.

1) CS have been shown to be elicited by unexpected perturbations during wrist movements in awake monkeys (Gilbert and Thach 1977), during skilled locomotion (Andersson and Armstrong 1987) and a step-like movement (Gellman et al. 1985) in awake cats, and during walking in decerebrate ferrets (Lou and Bloedel 1986, 1992). Because the cerebellum is involved in controlling both posture and movement, unexpected perturbations might be considered as errors (Oscarsson 1980) in postural performance and movement, because they imply a mismatch between desired and actual movement. In this connection it has been suggested that the mean firing rate of CS during several hundred milliseconds represents a sensory error signal (e.g., retinal slip). This hypothesis was derived from experimental data obtained in the rabbit flocculus during eye movements induced by movement of a large visual field (Graf et al. 1988; Simpson and Alley 1974). Furthermore, in the monkey VPFL during smooth pursuit eye movement induced by small target motion, transient retinal slip was shown to correlate with the occurrence of a single CS during steady-state pursuit (Stone and Lisberger 1990b). In Ojakangas and Ebner's study, CS was shown to be coupled to a velocity-related error signal during a voluntary arm movement (Ojakangas and Ebner 1994).

2) The CS have been suggested to be real-time motor commands that modulate SS (Mano et al. 1986) because for tens of milliseconds after a CS, there is a pause in SS firing (Bell and Grimm 1969) and/or there is a short-term modulation of SS discharges for several hundred milliseconds after CS firing (Ebner and Bloedel 1981).

3 ) The electrical coupling between IO neurons (Llinás et al. 1974; Sotelo et al. 1974) has been shown to cause a degree of CS synchrony among groups of Purkinje cells (Sugihara et al. 1993; Wylie et al. 1995). In the vestibulocerebellum in alert rabbit, CS synchrony was demonstrated during eye movement (De Zeeuw et al. 1997a). This characteristic, and the observation that $\mathrm{CS}$ have relatively rhythmic firing patterns (Welsh et al. 1995), has led to the suggestion that CS are phasic motor commands involved in controlling the timing of movement execution.

4) Results of physiological, anatomic, and behavioral studies, as well as the existence of long-term depression at parallel-fiber/Purkinje-cell synapses, support the proposal that the climbing fibers are involved in motor learning. In summary, SS provide motor commands that are regulated by CS via modulation of the efficacy of the parallel fiber inputs (Albus 1971; Ito 1984; Marr 1969).

Kawato and colleagues (Kawato and Gomi 1992a,b; Kawato et al. 1987) extended earlier learning models by formulating a computationally explicit feedback-error-learning model. In this model, CS are assumed to be copies of feedback motor commands generated by a crude feedback control circuit, and thus CS are suggested to be sensory error signals in motor coordinates. The model predicts that the cerebellar cortex acquires an inverse dynamics model of a controlled object as a result of this learning. There is an ongoing debate as to whether the plasticity at the parallel-fiber/Purkinje-cell synapse is the elementary process underlying motor adaptation and motor learning and what role climbing fibers may have in this process.

These hypotheses variously predict the information conveyed by CS, the relationship between SS and CS, and the function of CS. The information conveyed by CS is suggested to be either sensory error (hypotheses 1 and 4), motor commands ( hypothesis 2), or timing of movement (hypothesis 3 ). The feedback-error-learning model (Kawato and Gomi 1992b) predicts intermediate properties of CS, that is, that CS are derived from sensory error signals but already are represented temporally and spatially as feedback motor commands. CS and SS are suggested to be either independent (hypotheses 1 and 3 ) or related via short-term modulation (hypothesis 2) or long-term synaptic plasticity (hypothesis $4)$. The function of CS is suggested to involve either realtime motor control (hypotheses 2 and 3 ) or motor learning (hypothesis 4).

\section{Type of information encoded by CS and the mechanism involved}

Identification of the type of information and the mechanism by which that information is encoded by CS is of 
central importance in assessing the validity of the many current theories of climbing fiber function. CS are induced by vestibular (Amat 1983; Barmack et al. 1993; Ferin et al. 1971), visual (Fushiki et al. 1994; Graf et al. 1988; Maekawa and Simpson 1973; Simpson and Alley 1974; Stone and Lisberger 1990b), or other sensory stimuli. These data have been the basis of the common assumption that the climbing fibers convey sensory information to Purkinje cells. The present study adds new information about the motor characteristics of CS based on the following four quantitative analyses of CS firing while confirming that CS also have a sensory component.

First, the results of the present study indicate that the preferred directions of SS during OFR were close to either the vertical or horizontal axis. This is consistent with data obtained during smooth pursuit eye movements indicating that the preferred directions of SS in the VPFL were either ipsilateral or downward (Krauzlis and Lisberger 1996; Stone and Lisberger 1990a).

The preferred direction of SS and CS and their reference frame was extensively examined in the rabbit during optokinetic responses (OKR), using a 3-D planetarium projector (Graf et al. 1988). Because we did not use the 3-D planetarium projector stimulus system such as used in the rabbit's study (Graf et al. 1988), we could not answer the question about the spatial coordinates of CS or SS. However, Krauzlis and Lisberger (1996) suggested that the spatial coordinates of SS in the VPFL during smooth pursuit were aligned with the coordinates of either eye muscles or semicircular canals. Their argument was based on the following data. It has been shown in lateral and frontal eyed animals, that eye muscles and semicircular canals share a similar spatial reference frame (Graf and Simpson 1981). That is, the orientations of the planes of the horizontal, anterior and posterior semicircular canals are approximately aligned with the ipsilateral horizontal lecti, vertical lecti, and oblique muscles, respectively. The axes of eye muscles and semicircular canals of monkey are approximately vertical and approximately horizontal if they are projected in the front-parallel plane (Simpson et al. 1986).

In this study, because we fixed animal's head movement, sensory signals mainly came from visual system rather than from vestibular system. Furthermore, Shidara and Kawano (1993) reported that the preferred direction of SS of the Purkinje cells in the VPFL was either downward or ipsilateral, and at the site of each recording, electrical stimulation of the Purkinje cell with a single negative pulse elicited eye movement toward the preferred direction of the SS in more than one-half of the cases. Thus the spatial coordinate system of the SS is considered to be that of the motor commands or muscles rather than that of the vestibular input.

The medial superior temporal area of the cerebral cortex (MST) and the dorsolateral pontine nucleus (DLPN) provide visual information to the VPFL during OFR (Kawano et al. 1992, 1994a,b), and this pathway (MST-DLPNVPFL) has been suggested to be a major sensory-motor transformation circuit (Glickstein et al. 1985; Kawano et al. 1992, 1994a,b; Langer et al. 1985; Tusa and Ungerleider 1988). Most MST and DLPN neurons showed strong directional preferences for visual stimulus motion, and when their preferred directions (directions of movement associated with the most vigorous discharges) are plotted together in polar form, it is clear that all directions of motion are represented about equally (Kawano et al. 1992, 1994b). That is, the visual coordinates for OFR are uniformly distributed in all directions. On the other hand, the spatial coordinates of the extraocular motor neurons are segregated into three channels. Thus for OFR, the visual and motor coordinates are entirely different.

Consequently, the visual and motor components of CS can be evaluated by their spatial coordinate systems or, in other words, their preferred directions. Approximately opposite preferred directions of visual stimulus motion for CS and SS during OFR were observed in the present study, consistent with previous data, indicating that there is a reciprocal relation between SS and CS (De Zeeuw et al. 1995; Graf et al. 1988; Simpson and Alley 1974) and with data during smooth pursuit (Stone and Lisberger 1990b). The results of the present study clearly indicate that the spatial coordinates of CS during OFR were aligned with both SS and the motor command coordinates, suggesting that CS are represented in the motor-command coordinates.

Second, we examined the speed tuning properties of CS and SS during OFR. In rabbits, very low stimulus velocities $\left(<1^{\circ} / \mathrm{s}\right)$ are optimal for evoking CS (Simpson and Alley 1974), whereas in the monkey, CS can be evoked using higher stimulus velocities: $\leq 50^{\circ} / \mathrm{s}$ (Noda et al. 1987). We found that CS during OFR were evoked optimally at stimulus speeds of $40-80^{\circ} / \mathrm{s}$. The mean change in CS firing rate was more highly correlated with the mean eye velocity than with the mean retinal slip velocity. Furthermore, the mean change in CS firing rate was approximately a linear function of the eye velocity but was an approximately saturating sigmoid function of the retinal slip velocity. These results also suggest that CS firing patterns include a motor component. We used a much wider range of stimulus velocities than these in a previous study of smooth pursuit eye movement (Stone and Lisberger 1990b). In that study, because target speed was relatively low $\left(\sim 10^{\circ} / \mathrm{s}\right)$, the eye followed the step ramp motion of the stimuli quite well, and consequently the retinal slip was small. On the other hand, in our study, for the high speed stimuli, eye movement did not perfectly follow the step ramp motion, and the gains of the OFR were significantly lower than 1 (see Fig. 1, $A$ and $B$ ); consequently a large retinal slip remained even after the initiation of the OFR (see Fig. 6, $A, C$, and $F$ also). In our experiment, the temporal waveforms of the eye movement and the retinal slip were markedly different, so it was possible to examine sensory and motor components separately.

Third, we compared the SS and CS temporal firing profiles. Although the CS firing rate was very low and the CS temporal firing profile was opposite in polarity to that of SS, the CS firing probability was as accurately, or more accurately, reproduced from eye movement than that of the SS. The reciprocal relationship between SS and CS was supported quantitatively by the fact that their inverse dynamics coefficients were of opposite sign and the same order of magnitude. The onset of the SS and CS modulation preceded the onset of eye movement by a similar amount indicating that CS and SS were approximately synchronous. The estimated delay in the reconstruction study was close to that of the delay $(\sim 10 \mathrm{~ms})$ between the electrical stimulation of 
Purkinje cells in the VPFL and the resultant evoked eye movement (Shidara and Kawano 1993). In smooth pursuit eye movement, the modulation of SS and CS in the VPFL also is synchronized approximately, with a delay of $\sim 100$ $\mathrm{ms}$ from the onset of stimulus motion (Stone and Lisberger $1990 \mathrm{~b}$ ), which is $50 \mathrm{~ms}$ longer than that for OFR. It is interesting that CS and SS exhibit synchronous modulation in the different motor behaviors.

Fourth, further examination revealed that CS had comparatively larger eye acceleration components than SS. The firing rates of the MST and DLPN neurons, which provide visual inputs to the cerebellar cortex, are not well reproduced by applying the inverse dynamics model to the eye movements in response to a wide range of stimulus velocities (Kawano et al. 1994a). This supports the widely accepted idea that these two regions convey primarily sensory information. The velocity and acceleration coefficient ratio for the eye movement model for CS was 28 , which is closer to the ratio (33) for the MST and the ratio (12) for the DLPN (Takemura, Inoue, Kawano, Gomi, and Kawato, unpublished observations), suggesting that, as compared with SS, CS transmit information related more to visual inputs.

The data from the present study indicate that CS possess both sensory and motor aspects and cannot be characterized as purely sensory or purely motor in nature. Taken together, these findings indicate that the CS firing probability carries sensory error information derived from the retinal slip, but that it is already represented in the spatial and temporal frame of the dynamic motor commands. Because the Purkinje cells in the VPFL are synaptically distant from both the primary sensory and motor centers, it would a priori be very surprising if either SS or CS are purely sensory or motor in nature. The present analysis demonstrates that a more complex multimodal relationship must exist to account for the data.

\section{Real-time motor control by CS}

The results of the present study suggest the possibility that the motor component of CS includes real-time motor commands. We discuss conditions for this to work effectively.

First, the fact that the probability of CS firing can be accurately modeled using an inverse-dynamics representation indicates that the firing probability is extracted by either spatial or temporal averaging. The signal-to-noise ratio (mean divided by standard deviation: $p / \sqrt{p(1-p) / n}=$ $\sqrt{n p /(1-p)}$ of the firing rate improves in proportion to the square root of the trial number $n$ and the firing probability $p$. The rapid fluctuations in CS firing rate were relatively larger than those of SS because the CS firing probability is much smaller. There are three different ways to improve the signal-to-noise ratio for CS. First, because $p$ increases in proportion to the width of the time bin, a short time average (during several tens of milliseconds) improves the signalto-noise ratio while sacrificing the high temporal resolution signal. Second, if $n$ trials are averaged temporally, the firing probability can be extracted with an accuracy proportional to $\sqrt{n}$. A third method for improving the CS signal-to-noise ratio is by spatial averaging. In terms of real-time control functions of CS, those from many Purkinje cells may be summed and spatially averaged either in the brain stem or at extraocular motor neurons and the resulting signals may represent real-time motor commands. The fact that CS have very low firing rates speaks against this possibility, as discussed further in the following text. CS firing rates were not only very low (CS modulation was only $2-6 \%$ that of SS ) but were completely absent in many trials, and even when CS did occur, they consisted only of a single occurrence during the period of consideration. Thus although CS convey motor information, it appears unlikely that they contribute significantly to real-time motor control unless some strong nonlinear effects take place. For example, synchronization of the CS of many Purkinje cells may magnify the influence of CS on the downstream motor control system. However, the index of synchronization of the CS in pairs of Purkinje cells was shown to be quite small: only one order of magnitude larger than the value expected for a pair of random independent spike trains with similar firing rate of the CS (Sugihara et al. 1993). Thus for CS to function as effective real-time motor commands, it is essential to suppose that spatial averaging occurs and that some unknown strong nonlinear summation occurs in the brain stem.

Second, if, as generally supposed, SS convey the dynamic motor commands, then CS are of the opposite sign as motor commands because the modulations of CS frequency are of opposite sign of those of SS. In other words, CS in V cells are induced by upward stimulus motion, and if, like SS, they elicit downward eye movements, they would form a positive feedback loop that is harmful for real time control. The effect of CS on eye movement must, in fact, be upward if they are to form a real-time negative feedback loop to reduce the retinal slip. Three possible neural mechanisms to realize upward eye movement induced by CS will be discussed below. Direct short-term inhibition of SS by CS (Ebner and Bloedel 1981; Mano et al. 1986) is a possible sign inversion mechanism. However, in the spike trigger averaging analysis in Fig. $12 F$, we showed quantitatively that this effect was too weak compared with the direct effect of CS. Thus the short-term effect on SS does not change the polarity of the net effect of CS, because this is the summation of the direct effect of CS ( -2 to $-6 \%$ of SS) and the short-term pause in SS firing ( $1 \%$ of SS), which is still negative ( -1 to $-5 \%$; and very small). The same conclusion can be reached by directly calculating how many SS were suppressed by the short-term inhibition induced by a single CS. By integrating the SS-CS net cross-correlation of Fig. 12E, we estimate that only 0.11 SS were removed by a single CS ( $n=21$, in experiments 2 and 3 ). Thus the combined effect of the direct and short-term effects was $1-0.11=0.89$ of the original single CS and the resulting signal was still of the opposite sign of the motor command.

The second possibility is that because of the postinhibitory rebound burst (Llinás and Mühlethaler 1988), the net effect of CS on the recipient vestibular nuclei neurons could be an increase in firing, and CSs and SSs can be discriminated by the nuclei neurons, and the rebound excitation occurs only for CSs and not for SSs for some reasons which we do not understand. The third and most probable possibility is that the direct excitatory pathway from the IO to the cerebellar nucleus (De Zeeuw et al. 1997b; Sugihara et al. 1996) overrides the inhibitory effect of the CSs on the cerebellar nu- 
cleus. However, this argument implies that the cerebellar cortical pathway is not useful for on-line motor control although the IO itself is effective.

Finally, the CS temporal firing profile was too phasic (i.e., too acceleration-dependent; $B / M=28$ ) to be related to dynamic motor commands $(B / M=67)$. A signal must have the proper ratio of acceleration and velocity components of eye movement to function as a dynamic motor command.

In summary, the CS firing probability conveys high-frequency eye-movement-related information, and it may function as real-time motor commands during OFR, but only if some unknown strong nonlinear spatial summation of CS takes place and furthermore sign inversion occurs in the target cells only for CS (except for the short-term inhibition of SS by CS).

\section{Interactions between CS and SS for individual cells}

As discussed in the theory of CS function, possible interactions between CS and SS include short-term modulation and long-term synaptic plasticity. As discussed earlier, the short-term modulation was weak. In relation to long-term interactions, the following three aspects of the CS and SS firing characteristics were found to be negatively correlated on a cell-to-cell basis: the optimal stimulus direction from experiment 1 , the average amount of modulation from experiment 3 , and the temporal patterns of firing probability from experiments 2 and 3.

Four possible neural mechanisms that account for some of these correlations are as follows. First, the short-term inhibition of SS by CS may qualitatively explain all three correlations. As explained above, however, short-term inhibition was quantitatively weak, and only $1 \%$ of the SS modulation can be explained. Second, individual cell differences in size and/or input resistance may account for at least part of the second negative correlation but does not explain the first or third negative correlation. Third, some innate anatomic connectivity between mossy fiber inputs and climbing fiber inputs may explain general SS-CS reciprocity including the first negative correlation but does not explain the other two negative correlations. For example, if the climbing fiber inputs and the parallel fiber inputs have the same visual signal origin but the sign of one of them is inverted by an inhibitory relay center, then the general CS and SS reciprocity of directional selectivity and temporal firing profiles may be explained (Soodak et al. 1988). Because such connections are relatively nonspecific at a cell-to-cell level, however, they do not explain the specific cell-to-cell negative correlations (2nd and 3rd ones in preceding paragraph).

The fourth possible neural mechanism that may explain the three negative correlations is a long-term interaction between CS and SS. For the parallel-fiber/Purkinje-cell synapse, two types of synaptic plasticity in which the climbing fiber has a critical role are known: long-term depression, which occurs when the climbing fiber and the parallel fiber are simultaneously active (Ito 1989; Ito et al. 1982; Sakurai 1987), and long-term potentiation, which occurs when only the parallel fiber is active (Hirano 1991; Sakurai 1987). This bidirectional change (potentiation/depression) of synaptic efficacy may occur alternately for each parallel fiber synapse.
Then, on a cell-by-cell basis, this long-term interaction between CS and SS may lead to some reciprocity, that is, if the CS firing rate is high then the SS firing rate is low, and conversely if the CS firing rate is low then the SS firing rate is high for some specific characteristics of visual inputs. Thus at least qualitatively, it is possible that all three negative correlations can be explained by a long-term interaction between CS and SS.

Finally, we give a more computationally concrete model for these negative correlations based on previous theoretical studies (Kawato and Gomi 1992a,b). Suppose that the SS temporal firing profile of each cell is acquired through the temporal averaging of the CS temporal firing profile of the same cell based on synaptic plasticity. Equation 12 models the above process of long-term depression and potentiation, and Eqs. 13 and 14 its consequence for the SS temporal profile

$$
\begin{aligned}
\tau \mathrm{d} w_{i}(t) / \mathrm{d} t & =-\epsilon x_{i}(t)\left\{\mathrm{CS}(t)-\mathrm{CS}_{\text {spont }}\right\}-w_{i}(t) \\
\mathrm{SS}(t) & =\sum_{n} w_{i}(t) x_{i}(t) \\
& \simeq-\epsilon \sum_{n} \overline{x_{i}(t)\left\{\mathrm{CS}(t)-\mathrm{CS}_{\text {spont }}\right\}} x_{i}(t)
\end{aligned}
$$

where $w_{i}, x_{i}, \tau, \mathrm{SS}, \mathrm{CS}, \mathrm{CS}_{\text {spont }}$, and the overline, respectively denote the efficacy of the $i$ th parallel-fiber/Purkinjecell synapse, the firing rate of the $i$ th parallel fiber, the time constant of synaptic plasticity, the SS and CS firing rates, the spontaneous CS firing rate, and the temporal average. Assuming linear summation of synaptic inputs in SS (Eq. 13 ) and that time constant $\tau$ is much larger than the SS and CS temporal variations, Eq. 14 predicts that SS provide an approximate sign reversed temporal average of CS on a cellby-cell basis, because $w_{i}$ becomes positive, zero, and negative if $x_{i}(t)$ is negatively, not, and positively temporally correlated with CS $(t)-\mathrm{CS}_{\text {spont }}$. Considering the large cellto-cell variability in SS and CS firing characteristics, this temporal averaging leads directly to the cell-to-cell negative correlations between SS and CS. In this connection, we should consider the data in which the CSs were abolished by lesioning the inferior olive with lidocaine. The SS modulation remained, and the depth of SS modulation was unaffected (Leonard and Simpson 1986). This might be interpreted as compatible with the LTD-long-term potentiation mechanism described by Eq. 12 .

\section{CS may convey information sufficient for motor learning}

The two aspects of the experimental findings (cell-to-cell negative correlations and the sensory-motor nature of CS ) become a coherent picture if it is assumed, as above, that the SS temporal firing profile is acquired through the temporal averaging of CS based on synaptic plasticity as predicted in the cerebellar feedback-error-learning model (Kawato and Gomi 1992a,b). The feedback-error-learning model specifically proposes that CS provide a copy of crude feedback motor commands, which originally are derived from sensory signals and are used as an error signal in the acquisition of a cerebellar representation of the inverse model of a controlled object. The most unique aspect of this hypothesis is that the CS firing probability carries sensory error information derived from the retinal slip but already is represented in 
the spatial and temporal frame of the dynamic motor commands so that it can directly modify SS, thus predicting both the sensory and the motor characteristics of CS. Recent computational studies (Kawato and Gomi 1992a,b) revealed that this is the essential property of the error signal if it is to be directly used to modify the motor command based on a simple heterosynaptic plasticity rule such as long-term depression and potentiation [in more computational terms, the generalized $\delta$-rule (Rumelhart et al. 1986); WidrowHoff rule (Widrow-Hoff 1960); the least square-error method]. The following three specific predictions of the computational studies (Kawato and Gomi 1992a,b) were satisfied by the present data. The first prediction, that the spatial coordinate frame of CS is the same as that of SS was confirmed by the results of the directional tuning experiments. The second prediction was that the CS temporal firing profile is similar to that of SS; the reciprocal relationship between the SS and CS firing patterns has been shown previously in the low frequency range (De Zeeuw et al. 1995; Graf et al. 1988; Waespe and Henn 1981) and was demonstrated quantitatively in the present study in the high-frequency range (several hundreds of Hertz) as previously suggested (Keating and Thach 1995). The results of the present study also support the third prediction that CS encode error signals derived from the sensory signal but are already represented temporally and spatially in motor command coordinates.

It is interesting to consider why CS (the putative error signal) remain even after sufficient learning has presumably occurred. The system controlling OFR is a dynamic feedback control system with delay. In any feedback control system, the error cannot have an immediate influence on itself, because the controlled object has inertia and any feedback loop contains time delay. Thus the error cannot be suppressed to zero. This is obvious because, for example in OFR, the visual stimulus motion cannot be predicted beforehand and cannot be compensated for completely by the eye movement. Gomi and Kawato (1993) mathematically demonstrated that even without time delay the motor error signal is similar to the final motor command even after sufficient learning and thus is neither zero nor orthogonal to SS, the motor command. Time delays of $\sim 40,10$, and $50 \mathrm{~ms}$ exist between the stimulus motion onset and CS, between CS and eye movement, and between stimulus motion onset and eye movement, respectively. If the CS directly influences the SS $40 \mathrm{~ms}$ after the onset of stimulus motion through synaptic plasticity, then this change in SS leads to a change in eye movement (i.e., retinal slip) $10 \mathrm{~ms}$ later, $50 \mathrm{~ms}$ after the stimulus motion onset. The retinal slip then leads to a change in CS $40 \mathrm{~ms}$ later, $90 \mathrm{~ms}$ after the onset of stimulus motion. Thus a CS at any particular instant can, at the earliest, influence CS 50 ms after that instant and cannot be reduced to zero or be orthogonal to SS.

Although we needed to average discharges of $\mathrm{P}$ cells over many trials (and for some purpose over several cells), this is appropriate given the likelihood that such temporo-spatial averaging will be mimicked in vivo by spatial averaging occurring in the projections from Purkinje cells to floccular target neurons. There is a quantitative histological study of numerical data on cells and on synapses in the cerebellar nuclei in the cat (Palkovits et al. 1977). Probable conver- gence of Purkinje axons on nuclear cells are $\sim 860$, and the number of boutons of Purkinje cells origin is 11,600 per nuclear cell (Palkovits et al. 1977). These convergence may enable the target neurons to do spatial averaging.

The present study provides evidence as to why the CS firing rate is so low. Low membrane impedance due to gapjunctions in IO neurons may reduce the firing rate, but highfrequency information can be preserved via spatial averaging of many inputs by electrical coupling among the inferior olive neurons (Sotelo et al. 1974).

It appears that interference by CS in real-time movement control is minimized by their ultra-low firing rate, whereas the high-frequency information necessary for motor learning is preserved via neuronal temporal averaging (Eqs. 12-14). It is interesting to note that, in studies of adaptation, OFR gains were shown to change after a small number (tens) of trials (Miles and Kawano 1986), whereas the SS temporal firing profiles observed in the present study (Figs. 7 and 8) may be acquired over millions of OFRs. It remains the case that the system has not been studied while it is adapting. Miles and Kawano (1986) demonstrated that adaptation of OFR gain can be induced over the course of relatively few trials. By that experiment, we will be able to know directly how the CS will behave while they are most strongly exerting their proposed teaching function.

However, one significant factor in the present study is that perturbative "learning paradigms" such as causing the animal to learn or stimulating climbing fibers electrically were not used. Instead, we used analytic studies in normal behaving monkeys, the results of which suggest that CS represent sensory error signals that can directly modify the dynamic motor commands (SS) and reveal cell-to-cell negative correlations between the SS and CS firing characteristics, which are presumed to be acquired through life-long learning.

This work was supported by CREST-JST and Human Frontier Science Program.

Address for reprint requests: Y. Kobayashi, Dept. of Integrative Physiology, National Institute for Physiological Sciences, Myodaiji, Okazaki, Aichi 444-8585, Japan.

Received 29 December 1997; accepted in final form 3 April 1998.

\section{REFERENCES}

Albus, J. S. A theory of cerebellar function. Math Biosci. 10: 25-61, 1971. АмAт, J. Interaction between signals from vestibular forelimb receptors in Purkinje cells of the frog vestibulocerebellum. Brain Res. 278: 287-290, 1983.

Andersson, G. And Armstrong, D. M. Complex spikes in Purkinje cells in the lateral vermis of the cat cerebellum during locomotion. J. Physiol. (Lond.) 385: 107-134, 1987.

Barmack, N. H., Fagarson, M., Fredette, B. J., Mubnaini, E., And ShoJAKU, H. Activity of neurons in the beta nucleus of the inferior olive of the rabbit evoked by natural vestibular stimulation. Exp. Brain Res. 94: 203-215, 1993.

Bell, C. C. AND GRIMM, R. J. Discharge properties of Purkinje cells recorded on single and double micro electrodes. J. Neurophysiol. 32: 10441055, 1969.

De Zeeuw, C. I., Koekkoek, S.K.E., Wylie, D.R.W., and Simpson, J. I. Association between dendritic laminallar bodies and complex spike synchrony in the olivocerebellar system. J. Neurophysiol. 77: 1747-1758, 1997a.

De Zeeuw, C. I., Van Alphen, A. M., Hawkins, R. K., and Ruigrok, T. J. Climbing fiber collaterals contact neurons in the cerebellar nuclei 
that provide a GABAergic feedback to the inferior olive. Neuroscience 80: 981-986, 1997b.

De Zeeuw, C. I., Wylie, D.R.W., Stahl, J. S., and Simpson, J. I. Phase relations of Purkinje cells in the rabbit flocculus during compensatory movements. J. Neurophysiol. 74: 2051-2064, 1995.

EBNeR, T. J. AND BLOEDEL, J. R. Role of climbing fiber afferent input in determining climbing fiber responsiveness of Purkinje cells to mossy fiber input. J. Neurophysiol. 45: 962-971, 1981.

Ferin, M., Grigorian, R. A., and Strata, P. Mossy and climbing fiber activation in the cat cerebellum by stimulation of the labyrinth. Exp. Brain Res. 12: 1-17, 1971.

Fushiki, H., Sato, Y., Miura, A., And Kawasaki, T. Climbing fiber responses of Purkinje cells to retinal image movement in cat cerebellar flocculus. J. Neurophysiol. 71: 1336-1350, 1994.

Gellman, R., Gibson, A. R., AND Houk, J. C. Inferior olivary neurons in the awake cat: detection of contact and passive body displacement. $J$. Neurophysiol. 54: 40-60, 1985.

GERRITS, N. M. AND VoOGD, J. The climbing fiber projection to the flocculus and adjacent paraflocculus in the cat. Neuroscience 7: 2971-2991, 1982.

GERRITS, N. M. AND VoOGD, J. The topographical organization of climbing and mossy fiber afferents in flocculus and ventral paraflocculus in the rabbit, cat and monkey. Exp. Brain Res. 17, Suppl.: 26-29, 1989.

Gilbert, P.F.C. AND Thach, W. T. Purkinje cell activity during motor learning. Brain Res. 128: 309-328, 1977.

Glickstein, M., May, J., AND Mercer, B. E. Corticopontine projection in the macaque: the distribution of labeled cortical cells after large injections of horse-radish peroxidase in the pontine nuclei. J. Comp. Neurol. 235: $343-359,1985$.

Gomi, H. AND Kawato, M. Neural network control for a closed-loop system using feedback-error-learning. Neural Networks 6: 933-946, 1993.

Gomi, H., Shidara, M., Takemura, A., Inoue, Y., Kawano, K., and Kawato, M. Temporal firing patterns of Purkinje cells in the cerebellar ventral paraflocculus during ocular following responses in monkeys. I. Simple spikes. J. Neurophysiol. 80: 818-831, 1998.

GraF, W. AND Simpson, J. I. The relations between semicircular canals, the optic axis, and the extraocular muscle in lateral eyed and frontal eyed animals. In: Progress in Oculomotor Research Developments in Neuroscience, edited by A. Fucks and W. Becker. Amsterdam: Elsevier/ North-Holland, vol. 12, 1981, p. 411-420.

Graf, W., Simpson, J. I., AND LEONARD, C. S. Spatial organization of visual messages of the rabbit's cerebellar flocculus. II. Complex and simple spike responses of Purkinje cells. J. Neurophysiol. 60: 2091-2121, 1988.

HIRANO, T. Depression and potentiation of the synaptic transmission between a granule cell and Purkinje cell in rat cerebellar culture. Neurosci. Lett. 119: 141-144, 1991.

ITo, M. The Cerebellum and Neural Control. New York: Raven, 1984.

Iто, M. Long-term depression. Annu. Rev. Neurosci. 12: 85-102, 1989.

Ito, M., SAKURAI, M., AND TONGROACH, P. Climbing fiber induced depression of both mossy fiber responsiveness and glutamate sensitivity of cerebellar Purkinje cells. J. Physiol. (Lond.) 324: 113-134, 1982.

Kawano, K., Shidara, M., Takemura, A., Inoue, Y., Gomi, H., and Kawato, M. A linear time-series regression analysis of temporal firing patterns of cerebral, pontine and cerebellar neurons during the ocular following response. Jpn. J. Physiol. 44: 129, 1994a.

Kawano, K., Shidara, M., Takemura, A., Inoue Y., Gomi H., and KawATO, M. Inverse-dynamics representation of eye movements by cerebellar Purkinje cell activity during short-latency ocular-following responses. Ann. NY Acad. Sci. 781: 314-321, 1996.

Kawano, K., Shidara, M., Watanabe, Y., and Yamane, S. Neural activity in cortical area of MST of alert monkey during ocular following responses. J. Neurophysiol. 71: 2305-2324, 1994b.

Kawano, K., Shidara, M., AND Yamane, S. Neural activity in dorsolateral pontine nucleus of alert monkey during ocular following responses. $J$. Neurophysiol. 67: 680-703, 1992.

Kawato, M. Analysis of neural firing frequency by a generalized linear model. Tech. Rep. of IEICE NC95-33: 31-38, 1995.

Kawato, M., Furukawa, K., AND Suzuki, R. A. Hierarchical neural network model for control and learning of voluntary movement. Biol. Cybern. 57: 169-185, 1987.

Kawato, M. AND Gomi, H. The cerebellum and VOR/OKR learning models. Trends Neurosci. 15: 445-453, 1992a.

Kawato, M. AND Gomi, H. A computational model of four regions of the cerebellum based on feedback-error-learning. Biol. Cybern. 68: 95-103, 1992b.
Keating, J. G. AND Thach, W. T. Nonclock behavior of inferior olive neurons: interspike intervals of Purkinje cell complex spike discharge in the awake behaving monkey is random. J. Neurophysiol. 73: 1329-1340, 1995.

KeLLER, E. L. Accommodative vergence in the alert monkey motor unit analysis. Vision Res. 13: 1565-1575, 1973.

Kobayashi, Y., Kawano, K., and Kawato, M. The analysis of firing frequency of spike discharges using a generalized linear model (model examination). Tech. Rep. of IEICE NC95-34: 39-46, 1995.

KrauZlis, R. J. AND Lisberger, S. G. Directional organization of eye movement and visual signals in the floccular lobe of the monkey cerebellum. Exp. Brain Res. 109: 289-302, 1996.

Langer, T., Fuchs, A. F., Scudder, C. A., And Chubb, M. C. Afferents to the flocculus of the cerebellum in the rhesus macaque as revealed by retrograde transport of horseradish peroxidase. J. Comp. Neurol. 235: 125,1985

LeONARD, C. S. AND Simpson, J. I. Simple spike modulation of floccular Purkinje cells during the reversible blockage of their climbing fiber afferents. Adv. Biosci. 57: 321-328, 1986.

LlinÁs, R., BAKer, R., AND Sotelo, C. Electrotonic coupling between neurons in cat inferior olive. J. Neurophysiol. 37: 560-571, 1974.

LLINÁs, R. AND MUUHLETHALER, M. Electrophysiology of guinea-pig cerebellar nuclear cells in vitro brain stem-cerebellar preparation. J. Physiol. (Lond.) 404: 241-258, 1988.

LOU, J. S. AND BLOEDEL, J. R. The responses of simultaneously recorded Purkinje cells to the perturbations of the step cycle in the walking ferret: a study using a new analytical method-the real time post synaptic response (RTPR). Brain Res. 365: 340-344, 1986.

Lou, J. S. AND BLOEDEL, J. R. Responses of sagittally aligned Purkinje cells during perturbed locomotion: synchronous activation of climbing fiber inputs. J. Neurophysiol. 68: 570-580, 1992.

MaEKaWA, K. AND Simpson, J. I. Climbing fiber responses evoked in vestibulo-cerebellum of rabbit from visual system. J. Neurophysiol. 36: 649666, 1973.

Mano, N., Kanazawa, I., And Yamamoto, K. Complex-spike activity of cerebellar Purkinje cells related to wrist tracking movement in monkey. J. Neurophysiol. 56: 137-158, 1986.

MARr, D. A theory of cerebellar cortex. J. Physiol. (Lond.) 202: 437-470, 1969.

McCullagh, P. AND Nelder, F.R.S. Generalized Linear Models. London: Chapman Hall, 1989.

MiLes, F. A. AND Kawano, K. Short-latency ocular following responses of monkey. III. Plasticity. J. Neurophysiol. 56: 1381-1396, 1986.

Miles, F. A., Kawano, K., AND Optican, L. M. Short-latency ocular following responses of monkey. I. Dependence on temporospatial properties of visual input. J. Neurophysiol. 56: 1321-1354, 1986.

Noda, H., Warabi, T., AND OHNo, M. Response properties and visual receptive fields of climbing and mossy fibers terminating in the flocculus of the monkey. Exp. Neurol. 95: 455-471, 1987.

OJAKANGas, C. L. AND EBNER, T. J. Purkinje cell complex spike activity during voluntary motor learning: relationship to kinematics. J. Neurophysiol. 72: 2617-2630, 1994.

OsCARSSON, O. Functional organization of olivary projection to the cerebellar anterior lobe. In: The Inferior Olive Nucleus: Anatomy and Physiology, edited by J. Courvile, C. de Montigny, and Y. Lamarre. New York: Raven, 1980, p. 279-289.

Palkovits, M., Mezey, E., Hamori, J., and Szentagothai, J. Quantitative histological analysis of the cerebellar nuclei in the cat. I. Numerical data on cells and on synapses. Exp. Brain Res. 28: 189-209, 1977.

Perkel, D. H., Gerstein, G. L., AND Moore, G. P. Neuronal spike trains and stochastic point processes. II. Simultaneous spike train. Biophys. J. 7: 419-440, 1967.

Ron, S. And Robinson, D. A. Eye movements evoked by cerebellar stimulation in the alert monkey. J. Neurophysiol. 36: 1004-1022, 1973.

Rumelhart, D. E. and McClelland, J. L. Parallel Distributed Processing: Explorations in the Microstructure of Cognition, 1, 2. Cambridge, MA: MIT Press, 1986.

Rushmer, D. S., Roberts, W. J., AND Augter, G. K. Climbing fiber responses of cerebellar Purkinje cells to passive movement of the cat forepaw. Brain Res. 106: 1-20, 1976.

SAKURAI, M. Synaptic modification of parallel fiber-Purkinje cell transmission in vitro guinea-pig cerebellar slices. J. Physiol. (Lond.) 394: $463-$ 480, 1987.

Sato, Y., Miura, A., Fushiki, H., AND KawasAKI, T. Short-term modula- 
tion of cerebellar Purkinje cell activity after spontaneous climbing fiber input. J. Neurophysiol. 68: 2051-2062, 1992.

ShidARA, M. And Kawano, K. Role of Purkinje cells in the ventral paraflocculus in short-latency ocular following responses. Exp. Brain Res. 93: $185-195,1993$.

Shidara, M., Kawano, K., Gomi, H., and Kawato, M. Inverse-dynamics encoding of eye movements of Purkinje cells in the cerebellum. Nature 365: 50-52, 1993.

Simpson, J. I. AND Alley, K. E. Visual climbing fiber input to rabbit vestibulo-cerebellum: a source of direction-specific information. Brain Res. 82 302-308, 1974.

Simpson, J., Rudinger, D., Reisine, H., And Henn, V. Geometry of extraocular eye muscles of the rhesus monkey. Soc. Neurosci. Abstr. 12: 1186, 1986.

Simpson, J. I., Wylie, D. R., AND De Zeeuw, C. I. On climbing fiber signals and their consequence(s). Behav. Brain Sci. 19: 384-398, 1996.

Soodak, R. E., Croner, L. J., AND Graf, W. Development of the optokinetic reference frame of floccular Purkinje cells in rabbit. Soc. Neurosci. Abstr. 14: 758, 1988.

Sotelo, C., LlinÁs, R., AND BAKER, R. Structural study of inferior olivary nucleus of the cat: morphological correlates of electrotonic coupling. $J$. Neurophysiol. 37: 541-559, 1974.

Stone, L. S. And Lisberger, S. G. Visual responses of Purkinje cells in the cerebellar flocculus during smooth-pursuit eye movements in monkeys. I. Simple spikes. J. Neurophysiol. 63: 1241-1261, 1990a.

Stone, L. S. And Lisberger, S. G. Visual responses of Purkinje cells in the cerebellar flocculus during smooth-pursuit eye movements in monkeys. II. Complex spikes. J. Neurophysiol. 63: 1262-1275, 1990 b.

SugIHARA, I., LANG, E. J., AND LlinÁs, R. Uniform olivocerebellar conduction time underlies Purkinje cell complex spike synchronicity in the rat cerebellum. J. Physiol. (Lond.) 470: 243-271, 1993.

SugiharA, I., Wu, H., AND SHINODA, Y. Morphology of axon collateral of single climbing fibers in the deep cerebellar nuclei of the rat. Neurosci. Lett. 217: 33-36, 1996.

Тнасн, W. T. Discharge of cerebellar neurons during rapidly alternating arm movement in the monkey. J. Neurophysiol. 31: 785-797, 1968.

TOYAma, K., Kimura, M., And TANAKA, K. Cross-correlation analyses of interneuronal connectivity in cat visual cortex. J. Neurophysiol. 46: 191201, 1981.

TusA, R. J. AND UNGERLEIDER, L. G. Fiber pathways of cortical areas mediating smooth pursuit eye movements in monkeys. Ann. Neurol. 23: 174$183,1988$.

WAEsPe, W. AND HENN, V. Visual-vestibular interaction in the flocculus of the alert monkey. I. Input activity. Exp. Brain Res. 43: 349-360, 1981.

Welsh, J. P., Lang, J. E., Sugihara, I., and Llinas, R. Dynamic organization of motor control within the olivocerebellar system. Nature 374: $453-$ 457, 1995

Widrow, B. AND HoFf, M. E. Adaptive switching circuits. W ESCON Conv. Rec. IV: 96-104, 1960.

Wylie, D. R., DeZeeuw, C. I., And Simpson, J. I. Temporal relations of the complex spike activity of Purkinje cell pairs in the vestibulocerebellum of rabbits. J. Neurosci. 15: 2875-2887, 1995. 